Journal of

Mechanics of

Materials and Structures

NANOPARTICLES UNDER THE INFLUENCE OF SURFACE/INTERFACE ELASTICITY

Changwen $\mathrm{Mi}$ and Demitris A. Kouris

Volume 1, № 4

April 2006

mathematical sciences publishers 


\title{
NANOPARTICLES UNDER THE INFLUENCE OF SURFACE/INTERFACE ELASTICITY
}

\author{
Changwen Mi And Demitris A. Kouris
}

\begin{abstract}
We investigate the effect of surface/interface elasticity in the presence of nanoparticles, embedded in a semi-infinite elastic medium. The work is motivated by the technological significance of self-organization of strained islands in multilayered systems. Islands, adatom-clusters, or quantum dots are modeled as inhomogeneities, with properties that differ from the ones of the surrounding material. Within the framework of continuum elasticity theory, the Papkovitch-Neuber displacement methodology coupled with Gurtin's surface elasticity yields an analytical solution. The elastic field is expressed in terms of four sets of spherical and cylindrical harmonics. Surface elasticity introduces an additional length scale and results suggest that local stresses are significantly affected by the size of the nanoparticles.
\end{abstract}

\section{Introduction}

Self-assembly presents a promising alternative in fabrication of quantum dots. The main requirements for applications are size uniformity and high spatial density of dots. Currently, uniformity is on the order of $10 \%$ and no systematic approach has been proposed for its improvement. Covering of the first generation of islands or dots by a cap layer (say, of a substrate material) and growth of subsequent generations of dots result in the ultimate development of a two-dimensional superlattice. The spatial uniformity of island structures was first addressed by Tersoff et al. [1996]. Their islands were modeled as spherical inclusions (same properties as the surrounding material) in an elastic half-space, a problem that was originally solved by Mindlin and Cheng [1950]. Tersoff et al. [1996] chose to effectively treat the islands as point sources (centers of dilatation) in a semi-infinite matrix. In most material systems used in practice, however, quantum dots and the surrounding material do not have identical material properties.

The presence of inhomogeneities in elastic media has been a well studied subject in applied mechanics for several decades [Mura 1987]. Until recently, however, almost all such studies have been concerned with either an infinite medium or a semi-infinite body without including surface effects, either on the plane boundary,

Keywords: surface/interface effects, inhomogeneity, half-space. 
or at the matrix/inhomogeneity interface. Mindlin and Cheng [1950] first studied the thermoelastic stress in a semi-infinite solid containing a spherical inclusion subjected to dilatational thermal expansion. Tsuchida [1970; 1972] derived a threedimensional solution for displacements and stresses in a half-space having a spherical cavity, for various loading conditions. The classical solution for the elastic state of a spherical inhomogeneity in a semi-infinite elastic body without incorporating surface effects was obtained by Tsutsui et al. [1974].

These problems were solved within the context of classical elasticity, without accounting for size effects. It is well known, however, that under certain circumstances, surface elasticity can be of importance. For material structures with one or more dimensions at the nanometer scale (for example, thin film and nano-sized inhomogeneities), the surface area to volume ratio becomes significant. Consequently, surface effects have a major influence on the local stress-strain field.

The physical origin of the surface stress can be explained by the nature of the chemical bonding of those atoms close to the surface. The loss of the translational symmetry in the direction normal to surface results in a different bonding configuration in the vicinity of the surface, when compared to the one in the bulk. The atoms at and close to the surface lose some of their neighbors or are bonded to some "wrong" neighbors. As a result, the equilibrium interatomic distance for such atoms is different from the bulk atoms. This phenomenon results in an "excess" stress for atoms close to the surface.

The theory of isotropic surface elasticity was established by Gurtin and Murdoch [1975a; 1975b; 1978]. Cahn and Larche [1982] studied the mechanical and chemical equilibrium of a small spherical precipitate in an infinite matrix of a different phase. Recently Sharma et al. [2003] derived closed-form expressions for the displacements and stresses of a strained spherical inhomogeneity by using a variational approach. Sharma and Ganti [2004] also studied the elastic state of spherical and cylindrical inhomogeneities embedded in an infinite elastic medium, by combining the classical Green's function method with linear isotropic surface elasticity theory. Duan et al. [2005b] developed explicit forms of the stress concentration tensor for spherical and circular inhomogeneities with surface effects. While surface/interface effects were incorporated, all of these investigations have been limited to the study of an infinite elastic medium.

In the present work, we model a nanoparticle or island as a spherical inhomogeneity problem inside an elastic half-space. The material properties of the inhomogeneity are different from those of the surrounding material (matrix). The system is subjected to biaxial tension applied at the remote boundary of the matrix, parallel to the free surface. The ensuing three-dimensional problem is solved with the help of the Papkovitch-Neuber displacement potential theory. Section 2 illustrates the formulation of the problem of an embedded inhomogeneity in a half-space including 
surface/interface effects. Following [Gurtin et al. 1998], the displacements on the surface/interface boundary are still considered to be continuous. The tractions both on the plane boundary and at the matrix/inhomogeneity interface, however, are discontinuous, as a result of the well known Laplace-Young effects [Adamson 1982, Chapter II]. The problem is solved in Section 3, incorporating the surface/interface stress boundary conditions. Section 4 illustrates the impact of surface/interface effects as the location, size, and material properties of the inhomogeneity change. Finally, a series of conclusions are presented in Section 5. The elaborate and quite tedious mathematical formulation presented in Sections 2 and 3 is the result of a conscious decision to provide a complete, self-contained document, since the detailed methodology cannot be found elsewhere.

\section{Displacement formulation}

Let us consider a spherical inhomogeneity in a semi-infinite elastic solid, as shown in Figure 1. The center of the inhomogeneity coincides with the center of the coordinate system. Cylindrical $(r, \theta, z)$ and spherical $(R, \theta, \varphi)$ coordinates will be used alternatively throughout the manuscript. Following [Gurtin and Murdoch 1978], the plane boundary $(z=-c)$ and the matrix/inhomogeneity interface $(R=a)$ are modeled as thin films of vanishing depth; both "surfaces" adhere to the bulk without slipping. The model is based on the assumption of a linearly elastic and isotropic material system.

The governing equations for the bulk are given by

$$
\sigma_{i j, j}=0, \quad \sigma_{i j}=2 G\left(\frac{v}{1-2 v} \varepsilon_{k k} \delta_{i j}+\varepsilon_{i j}\right), \quad \varepsilon_{i j}=\frac{1}{2}\left(u_{i, j}+u_{j, i}\right),
$$

coupled with the equations for surfaces and interfaces derived in [Gurtin and Murdoch 1975a; 1975b]

$$
\begin{aligned}
\varepsilon_{\alpha \beta}^{S} & =\frac{1}{2}\left(P_{i j} \varepsilon_{j k} P_{k l}+P_{i j} \bar{\varepsilon}_{j k} P_{k l}\right), \\
\sigma_{\alpha \beta}^{S} & =\tau_{0} \delta_{\alpha \beta}+2\left(\mu^{S}-\tau_{0}\right) \varepsilon_{\alpha \beta}^{S}+\left(\lambda^{S}+\tau_{0}\right) \varepsilon_{\kappa \kappa}^{S} \delta_{\alpha \beta}+\tau_{0}\left(u_{\alpha}^{S}\right)_{, \beta}^{S}, \\
{\left[\sigma_{i j}\right] n_{j} } & =-\left(\sigma_{\alpha \beta}^{S}\right)_{, \beta}^{S} .
\end{aligned}
$$

By convention, the Roman subscripts denote the quantities belonging to the bulk and assume values from 1 to 3 , while the Greek refer to the surface/interface and assume values from 1 to 2 . Here $u, \varepsilon$ and $\sigma$ denote displacement, strain and stress fields in bulk; the material properties of the bulk are represented by the shear modulus $G$ and Poisson's ratio $\nu ; \lambda^{S}, \mu^{S}$ and $\tau_{0}$ refer to the surface Lamé constants, and the residual surface stress when the bulk is unstrained, respectively; $\left[\sigma_{i j}\right]=\sigma_{i j}$ (out) $-\sigma_{i j}$ (in) and $n$ is the outward unit normal to the surface; $\varepsilon^{S}$ and $\sigma^{S}$ denote the surface strain and surface stress fields on the surface. Since 

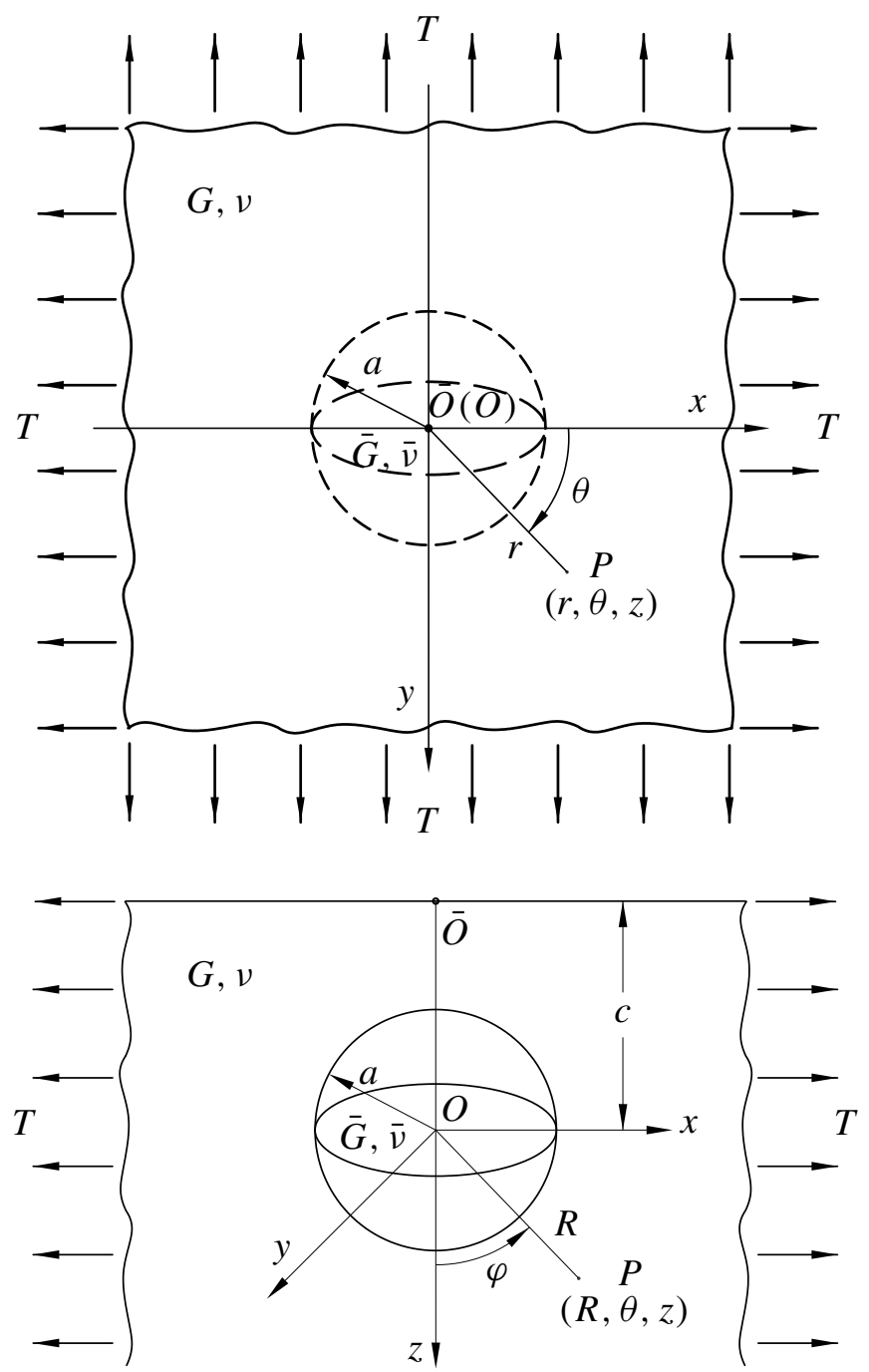

Figure 1. Spherical inhomogeneity in a half-space.

the surface is defined as a two-dimensional continuous space, the components of surface strain and surface stress with direction normal to the surface vanish. $P_{i j}=\delta_{i j}-n_{i} n_{j}$ is the projection tensor which allows tensor transformation between the three-dimensional bulk space and the two-dimensional surface space. In terms of the projection tensor, the surface strain is defined as the average value of the bulk strains from both sides projected onto the surface. The notion of surface gradient, surface divergence of a vector, and surface divergence of a superficial tensor can 
be clarified by the following identities (see [Gurtin et al. 1998]):

$$
\left(u_{\alpha}^{S}\right)_{, \beta}^{S}=u_{i, k} P_{k j}, \quad a_{\alpha}\left(\alpha_{\alpha \beta}^{S}\right)_{, \beta}^{S}=\left(\sigma_{\beta \alpha}^{S} \alpha_{\beta}\right)_{, \alpha}^{S}=\operatorname{tr}\left(\left(\sigma_{\beta \alpha}^{S} \alpha_{\beta}\right)_{, \gamma}^{S}\right),
$$

for every constant vector $a$, where tr is the trace. For convenience, the surface divergence of the surface stress tensor will be denoted by $\gamma$.

Both in the embedded particle and surrounding matrix, the displacement field satisfies the following Navier's equation (without body forces):

$$
\frac{1}{1-2 v} u_{j, j i}+u_{i, j j}=0 \text {. }
$$

In the case of axial symmetry without torsion, its solution can be expressed as superposition of two displacement fields due to potentials $\phi_{0}$ and $\phi_{3}$. In cylindrical coordinates, these displacements and the corresponding strains and stresses are given by

$$
\begin{aligned}
2 G u_{r} & =\frac{\partial \phi_{0}}{\partial r}+z \frac{\partial \phi_{3}}{\partial r}, \\
2 G u_{z} & =\frac{\partial \phi_{0}}{\partial z}+z \frac{\partial \phi_{3}}{\partial z}-(3-4 v) \phi_{3}, \\
2 G \varepsilon_{r r} & =-\frac{1}{r} \frac{\partial \phi_{0}}{\partial r}-\frac{\partial^{2} \phi_{0}}{\partial z^{2}}-\frac{z}{r} \frac{\partial \phi_{3}}{\partial r}-z \frac{\partial^{2} \phi_{3}}{\partial z^{2}}, \\
2 G \varepsilon_{\theta \theta} & =\frac{1}{r} \frac{\partial \phi_{0}}{\partial r}+\frac{z}{r} \frac{\partial \phi_{3}}{\partial r}, \\
2 G \varepsilon_{z z} & =\frac{\partial^{2} \phi_{0}}{\partial z^{2}}-2(1-2 v) \frac{\partial \phi_{3}}{\partial z}+z \frac{\partial^{2} \phi_{3}}{\partial z^{2}}, \\
2 G \varepsilon_{r z} & =\frac{\partial^{2} \phi_{0}}{\partial r \partial z}-(1-2 v) \frac{\partial \phi_{3}}{\partial r}+z \frac{\partial^{2} \phi_{3}}{\partial r \partial z}, \\
\sigma_{r r} & =-\frac{1}{r} \frac{\partial \phi_{0}}{\partial r}-\frac{\partial^{2} \phi_{0}}{\partial z^{2}}-\frac{z}{r} \frac{\partial \phi_{3}}{\partial r}-z \frac{\partial^{2} \phi_{3}}{\partial z^{2}}-2 v \frac{\partial \phi_{3}}{\partial z}, \\
\sigma_{\theta \theta} & =\frac{1}{r} \frac{\partial \phi_{0}}{\partial r}+\frac{z}{r} \frac{\partial \phi_{3}}{\partial r}-2 v \frac{\partial \phi_{3}}{\partial z}, \\
\sigma_{z z} & =\frac{\partial^{2} \phi_{0}}{\partial z^{2}}-2(1-v) \frac{\partial \phi_{3}}{\partial z}+z \frac{\partial^{2} \phi_{3}}{\partial z^{2}}, \\
\sigma_{r z} & =\frac{\partial^{2} \phi_{0}}{\partial r \partial z}-(1-2 v) \frac{\partial \phi_{3}}{\partial r}+z \frac{\partial^{2} \phi_{3}}{\partial r \partial z}, \\
u_{\theta} & 0, \quad \varepsilon_{r \theta}=\varepsilon_{\theta z}=0, \sigma_{\theta z}=0,
\end{aligned}
$$


where $\nabla^{2} \phi_{0}=\nabla^{2} \phi_{3}=0$ and $\nabla^{2}=\frac{\partial^{2}}{\partial r^{2}}+\frac{1}{r} \frac{\partial}{\partial r}+\frac{\partial^{2}}{\partial z^{2}}$. Converting these equations to spherical coordinates via

$$
z=R \cos \varphi \quad \text { and } \quad r=R \sin \varphi
$$

we get for the spherical components of displacements, strains and stresses

$$
\begin{aligned}
& 2 G u_{R}=\frac{\partial \phi_{0}}{\partial R}+\mu\left(R \frac{\partial \phi_{3}}{\partial R}-(3-4 v) \phi_{3}\right), \\
& 2 G u_{\varphi}=\sqrt{1-\mu^{2}}\left(-\frac{1}{R} \frac{\partial \phi_{0}}{\partial \mu}-\mu \frac{\partial \phi_{3}}{\partial \mu}+(3-4 v) \phi_{3}\right), \\
& 2 G \varepsilon_{R R}=\frac{\partial^{2} \phi_{0}}{\partial R^{2}}+\mu\left(R \frac{\partial^{2} \phi_{3}}{\partial R^{2}}-2(1-2 v) \frac{\partial \phi_{3}}{\partial R}\right), \\
& 2 G \varepsilon_{\theta \theta}=\frac{1}{R}\left(\frac{\partial \phi_{0}}{\partial R}-\frac{\mu}{R} \frac{\partial \phi_{0}}{\partial \mu}\right)+\mu\left(\frac{\partial \phi_{3}}{\partial R}-\frac{\mu}{R} \frac{\partial \phi_{3}}{\partial \mu}\right), \\
& 2 G \varepsilon_{\varphi \varphi}=-\frac{\partial^{2} \phi_{0}}{\partial R^{2}}-\frac{1}{R} \frac{\partial \phi_{0}}{\partial R}+\frac{\mu}{R^{2}} \frac{\partial \phi_{0}}{\partial \mu}-R \mu \frac{\partial^{2} \phi_{3}}{\partial R^{2}}-\mu \frac{\partial \phi_{3}}{\partial R} \\
& +\frac{\left(-2(1-2 v)+(3-4 v) \mu^{2}\right)}{R} \frac{\partial \phi_{3}}{\partial \mu}, \\
& 2 G \varepsilon_{R \varphi}=\sqrt{1-\mu^{2}} \\
& \times\left(\frac{1}{R^{2}} \frac{\partial \phi_{0}}{\partial \mu}-\frac{1}{R} \frac{\partial^{2} \phi_{0}}{\partial R \partial \mu}+(1-2 v) \frac{\partial \phi_{3}}{\partial R}-\mu \frac{\partial^{2} \phi_{3}}{\partial R \partial \mu}+\frac{2(1-v) \mu}{R} \frac{\partial \phi_{3}}{\partial \mu}\right), \\
& \sigma_{R R}=\frac{\partial^{2} \phi_{0}}{\partial R^{2}}+R \mu \frac{\partial^{2} \phi_{3}}{\partial R^{2}}-2(1-v) \mu \frac{\partial \phi_{3}}{\partial R}-\frac{2 v\left(1-\mu^{2}\right)}{R} \frac{\partial \phi_{3}}{\partial \mu}, \\
& \sigma_{\theta \theta}=\frac{1}{R}\left(\frac{\partial \phi_{0}}{\partial R}-\frac{\mu}{R} \frac{\partial \phi_{0}}{\partial \mu}\right)+(1-2 v) \mu \frac{\partial \phi_{3}}{\partial R}-\frac{\left(\mu^{2}(1-2 v)+2 v\right)}{R} \frac{\partial \phi_{3}}{\partial \mu}, \\
& \sigma_{\varphi \varphi}=-\frac{\partial^{2} \phi_{0}}{\partial R^{2}}-\frac{1}{R} \frac{\partial \phi_{0}}{\partial R}+\frac{\mu}{R^{2}} \frac{\partial \phi_{0}}{\partial \mu}-R \mu \frac{\partial^{2} \phi_{3}}{\partial R^{2}}-(1+2 \nu) \mu \frac{\partial \phi_{3}}{\partial R} \\
& +\frac{(3-2 v) \mu^{2}-2(1-v)}{R} \frac{\partial \phi_{3}}{\partial \mu}, \\
& \sigma_{R \varphi}=\sqrt{1-\mu^{2}}\left(\frac{1}{R^{2}} \frac{\partial \phi_{0}}{\partial \mu}-\frac{1}{R} \frac{\partial^{2} \phi_{0}}{\partial R \partial \mu}+(1-2 v) \frac{\partial \phi_{3}}{\partial R}-\mu \frac{\partial^{2} \phi_{3}}{\partial R \partial \mu}+\frac{2(1-v) \mu}{R} \frac{\partial \phi_{3}}{\partial \mu}\right), \\
& u_{\theta}=0, \quad 2 G \varepsilon_{R \theta}=2 G \varepsilon_{\theta \varphi}=0, \quad \sigma_{R \theta}=\sigma_{\theta \varphi}=0,
\end{aligned}
$$


where $\mu=\cos \varphi$, and the harmonic operator now takes the form

$$
\nabla^{2}=\frac{\partial^{2}}{\partial R^{2}}+\frac{2}{R} \frac{\partial}{\partial R}-\frac{2 \mu}{R^{2}} \frac{\partial}{\partial \mu}+\frac{\left(1-\mu^{2}\right)}{R^{2}} \frac{\partial^{2}}{\partial \mu^{2}} .
$$

For the purpose of completeness, we now derive general expressions for surface/interface quantities, both on the plane boundary $(z=-c)$ and on the matrix/inhomogeneity interface $(R=a)$. In cylindrical coordinates, the projection tensor for the plane boundary is given by

$$
P_{r r}=P_{\theta \theta}=1, \quad \text { all other } P_{i j}=0 .
$$

Substituting Equations (3) and (5) into the first equation of (1), and the first equation of (2), the surface strain tensor and the surface gradient of the surface displacement on the plane boundary are found to be

$$
\begin{aligned}
\varepsilon_{r r}^{f s} & =u_{r, r}^{f s}=-\frac{1}{4 G}\left(\frac{1}{r} \frac{\partial \phi_{0}}{\partial r}+\frac{\partial^{2} \phi_{0}}{\partial z^{2}}+\frac{z}{r} \frac{\partial \phi_{3}}{\partial r}+z \frac{\partial^{2} \phi_{3}}{\partial z^{2}}\right)_{z=-c}, \\
\varepsilon_{\theta \theta}^{f s} & =u_{\theta, \theta}^{f s}=\frac{1}{4 G}\left(\frac{1}{r} \frac{\partial \phi_{0}}{\partial r}+\frac{z}{r} \frac{\partial \phi_{3}}{\partial r}\right)_{z=-c}, \\
\varepsilon_{r \theta}^{f s} & =u_{r, \theta}^{f s}=0,
\end{aligned}
$$

where the superscript $f$ denotes the plane boundary. By the use of these relations and the second equation of (1), it is found that

$$
\begin{aligned}
\sigma_{r r}^{f s} & =\tau_{f 0}-\left(\chi_{f 2} \frac{1}{r} \frac{\partial \phi_{0}}{\partial r}+\chi_{f 0} \frac{\partial^{2} \phi_{0}}{\partial z^{2}}+\chi_{f 2} \frac{z}{r} \frac{\partial \phi_{3}}{\partial r}+\chi_{f 0} z \frac{\partial^{2} \phi_{3}}{\partial z^{2}}\right)_{z=-c}, \\
\sigma_{\theta \theta}^{f s} & =\tau_{f 0}+\left(\chi_{f 2} \frac{1}{r} \frac{\partial \phi_{0}}{\partial r}-\chi_{f 1} \frac{\partial^{2} \phi_{0}}{\partial z^{2}}+\chi_{f 2} \frac{z}{r} \frac{\partial \phi_{3}}{\partial r}-\chi_{f 1} z \frac{\partial^{2} \phi_{3}}{\partial z^{2}}\right)_{z=-c}, \\
\sigma_{r \theta}^{f s} & =0,
\end{aligned}
$$

where $\tau_{f 0}$ denotes the deformation-independent surface stress defined on the plane boundary. The length scale parameters $\chi_{f 0}, \chi_{f 1}$, and $\chi_{f 2}$ are defined in Equation (28) in the Appendix. The surface divergence of the surface stress tensor can be obtained by transforming the second equation of (2) into cylindrical coordinates. An explicit formula for evaluating this vector can be found in [Duan et al. 2005a, (2.2)]. As a result, the surface divergence of the surface stress $\gamma^{f}$ on the plane boundary is given by

$$
\gamma_{r}^{f}=-\chi_{f 0}\left(\frac{\partial^{3} \phi_{0}}{\partial r \partial z^{2}}+z \frac{\partial^{3} \phi_{3}}{\partial r \partial z^{2}}\right)_{z=-c}, \quad \gamma_{\theta}^{f}=\gamma_{z}^{f}=0 .
$$

For the matrix/inhomogeneity interface $(R=a)$, the projection tensor is

$$
P_{\theta \theta}=P_{\varphi \varphi}=1, \quad \text { all other } P_{i j}=0 .
$$


The relevant interface quantities can be derived from this equation together with (1), (2) and (4):

$$
\begin{aligned}
& \varepsilon_{\theta \theta}^{s}=u_{\theta, \theta}^{s}=\frac{1}{4 G}\left(\frac{1}{R} \frac{\partial \phi_{0}}{\partial R}-\frac{\mu}{R^{2}} \frac{\partial \phi_{0}}{\partial \mu}+\mu \frac{\partial \phi_{3}}{\partial R}-\frac{\mu^{2}}{R} \frac{\partial \phi_{3}}{\partial \mu}\right)_{R=a}, \\
& \varepsilon_{\varphi \varphi}^{s}=u_{\theta, \theta}^{s}=\frac{1}{4 G}\left(\frac{\mu}{R^{2}} \frac{\partial \phi_{0}}{\partial \mu}-\frac{\partial^{2} \phi_{0}}{\partial R^{2}}-\frac{1}{R} \frac{\partial \phi_{0}}{\partial R}-R \mu \frac{\partial^{2} \phi_{3}}{\partial R^{2}}-\mu \frac{\partial \phi_{3}}{\partial R}\right.\left.+\frac{-2(1-2 v)+(3-4 v) \mu^{2}}{R} \frac{\partial \phi_{3}}{\partial \mu}\right)_{R=a} \\
& \varepsilon_{\theta \varphi}^{s}=u_{\theta, \varphi}^{s}=0,
\end{aligned}
$$

$$
\begin{gathered}
\sigma_{\theta \theta}^{s}=\tau_{0}+\left(-\chi_{1} \frac{\partial^{2} \phi_{0}}{\partial R^{2}}+\chi_{2} \frac{1}{R} \frac{\partial \phi_{0}}{\partial R}-\chi_{2} \frac{\mu}{R^{2}} \frac{\partial \phi_{0}}{\partial \mu}-\chi_{1} R \mu \frac{\partial^{2} \phi_{3}}{\partial R^{2}}+\chi_{2} \mu \frac{\partial \phi_{3}}{\partial R}\right. \\
\left.-\frac{2(1-2 v) \chi_{1}+\left(\chi_{0}-(3-4 v) \chi_{1}\right) \mu^{2}}{R} \frac{\partial \phi_{3}}{\partial \mu}\right)_{R=a}
\end{gathered}
$$$$
\sigma_{\varphi \varphi}^{s}=\tau_{0}+\left(-\chi_{0} \frac{\partial^{2} \phi_{0}}{\partial R^{2}}-\chi_{2} \frac{1}{R} \frac{\partial \phi_{0}}{\partial R}+\chi_{2} \frac{\mu}{R^{2}} \frac{\partial \phi_{0}}{\partial \mu}-\chi_{0} R \mu \frac{\partial^{2} \phi_{3}}{\partial R^{2}}-\chi_{2} \mu \frac{\partial \phi_{3}}{\partial R}\right.
$$

$$
\left.+\frac{-2(1-2 v) \chi_{0}+\left((3-4 v) \chi_{0}-\chi_{1}\right) \mu^{2}}{R} \frac{\partial \phi_{3}}{\partial \mu}\right)_{R=a},
$$

$\sigma_{\theta \varphi}^{s}=0$,

$$
\begin{aligned}
& \gamma_{R}=\left(-\frac{2 \tau_{0}}{R}+\frac{\chi_{3}}{R} \frac{\partial^{2} \phi_{0}}{\partial R^{2}}+\chi_{3} \mu \frac{\partial^{2} \phi_{3}}{\partial R^{2}}+\frac{2(1-v) \chi_{3}\left(1-\mu^{2}\right)}{R^{2}} \frac{\partial \phi_{3}}{\partial \mu}\right)_{R=a}, \\
& \gamma_{\theta}=0
\end{aligned}
$$$$
\gamma_{\varphi}=\sqrt{1-\mu^{2}}\left(\frac{\partial}{\partial \mu}\left(\frac{\chi_{0}}{R} \frac{\partial^{2} \phi_{0}}{\partial R^{2}}+\frac{\chi_{2}}{R^{2}} \frac{\partial \phi_{0}}{\partial R}-\frac{\chi_{2}}{R^{3}} \phi_{0}\right)\right.
$$$$
\begin{aligned}
& +\mu \frac{\partial}{\partial \mu}\left(\chi_{0} \frac{\partial^{2} \phi_{3}}{\partial R^{2}}+\frac{\chi_{2}}{R} \frac{\partial \phi_{3}}{\partial R}-\frac{4(1-v) \chi_{2}}{R^{2}} \phi_{3}\right) \\
& \left.\quad-\left((1-4 v) \chi_{0} \frac{\partial^{2} \phi_{3}}{\partial R^{2}}+\frac{(3-8 v) \chi_{0}+\chi_{1}}{R} \frac{\partial \phi_{3}}{\partial R}\right)\right)_{R=a},
\end{aligned}
$$

where $\tau_{0}$ is the deformation-independent interface stress defined at the spherical interface. The interface length scale parameters $\chi_{0}, \chi_{1}, \chi_{2}$, and $\chi_{3}$ are defined in Equation (29) of the Appendix.

Given the expressions for the surface divergence of the surface stress (7), the boundary conditions at the free surface can be expressed in cylindrical coordinates 
as

$$
\left(\sigma_{r z}\right)_{z=-c}=-\gamma_{r}^{f}, \quad\left(\sigma_{z z}\right)_{z=-c}=0 .
$$

At infinity,

$$
\left(\sigma_{r r}\right)_{r \rightarrow \infty}=\left(\sigma_{\theta \theta}\right)_{r \rightarrow \infty}=T, \quad\left(\sigma_{r z}\right)_{r \rightarrow \infty}=\left(\sigma_{z z}\right)_{r \rightarrow \infty}=0,
$$

corresponding to the far-field biaxial tension. The boundary conditions at the matrix/inhomogeneity interface consist of the displacement continuity condition and the third equation in (1). These can be rewritten as follows, using Equations (8) for the $\gamma$ 's:

$$
\begin{aligned}
\left(u_{R}\right)_{R=a}=\left(\bar{u}_{R}\right)_{R=a}, & \left(u_{\varphi}\right)_{R=a}=\left(\bar{u}_{\varphi}\right)_{R=a}, \\
\left(\sigma_{R R}\right)_{R=a}-\left(\bar{\sigma}_{R R}\right)_{R=a}=-\gamma_{R}, & \left(\sigma_{R \varphi}\right)_{R=a}-\left(\bar{\sigma}_{R \varphi}\right)_{R=a}=-\gamma_{\varphi},
\end{aligned}
$$

where the quantities denoted by an over bar refer to the inhomogeneity.

In cylindrical and spherical coordinates, the general solution to the harmonic equation can be expressed in terms of cylindrical or spherical harmonics, respectively. Based on these basic solutions, four sets of displacement potentials are selected to represent the solutions to our problem. The first represents the biaxial tension applied at infinity and is given by

$$
\frac{\phi_{0}}{T}=-\frac{(1-v)}{(1+v)} R^{2} P_{2}(\mu), \quad \frac{\phi_{3}}{T}=-\frac{1}{(1+v)} R P_{1}(\mu) .
$$

Here $P_{n}(\mu)$ is the Legendre function of the first kind of order $n$, with $P_{0}(\mu)=1$, $P_{1}(\mu)=\mu, P_{2}(\mu)=\left(3 \mu^{2}-1\right) / 2$, and so forth. In terms of cylindrical coordinates, (12) can be rewritten as

$$
\frac{\phi_{0}}{T}=-\frac{(1-v)}{2(1+v)}\left(2 z^{2}-r^{2}\right), \quad \frac{\phi_{3}}{T}=-\frac{1}{(1+v)} z .
$$

The disturbance due to the presence of the inhomogeneity can be expressed by the following three sets of displacement potentials:

$$
\begin{gathered}
\frac{\phi_{0}}{T}=\sum_{n=0}^{\infty} A_{n} \frac{P_{n}(\mu)}{R^{n+1}}, \quad \frac{\phi_{3}}{T}=\sum_{n=0}^{\infty} B_{n} \frac{P_{n}(\mu)}{R^{n+1}} ; \\
\frac{\phi_{0}}{T}=\int_{0}^{\infty} \psi_{1}(\lambda) J_{0}(\lambda r) e^{-\lambda z} d \lambda, \quad \frac{\phi_{3}}{T}=\int_{0}^{\infty} \lambda \psi_{2}(\lambda) J_{0}(\lambda r) e^{-\lambda z} d \lambda
\end{gathered}
$$

for the matrix (with $z>-c, R>a$ ), and

$$
\frac{\phi_{0}}{T}=\sum_{n=0}^{\infty} \bar{A}_{n} R^{n} P_{n}(\mu), \quad \frac{\phi_{3}}{T}=\sum_{n=0}^{\infty} \bar{B}_{n} R^{n} P_{n}(\mu)
$$


for the inhomogeneity $(R<a)$. In these expressions, $J_{v}(\lambda r)$ is the Bessel function of the first kind of order $v ; A_{n}, B_{n}, \bar{A}_{n}$ and $\bar{B}_{n}$ are unknown coefficients of the spherical harmonics; and $\psi_{1}(\lambda)$ and $\psi_{2}(\lambda)$ are unknown functions of the integral variable $\lambda$. With these choices of the displacement potentials, the boundary condition (10) is satisfied automatically. The unknown constants $A_{n}, B_{n}, \bar{A}_{n}, \bar{B}_{n}$ and the unknown functions $\psi_{1}(\lambda), \psi_{2}(\lambda)$ are to be determined by enforcing the boundary conditions (9) and (11).

\section{Solution of the problem}

The displacement potential (13) is expressed in spherical coordinates. In order to satisfy the boundary condition (9), it must be transformed into cylindrical coordinates. From [Sneddon [1951], p.514], it is found that for $z \geq 0$,

$$
\frac{P_{n}(\mu)}{R^{n+1}}=\frac{1}{n !} \int_{0}^{\infty} \lambda^{n} J_{0}(\lambda r) e^{-\lambda z} d \lambda
$$

Replacing $(z)$ with $(-z),(\mu)$ with $(-\mu)$, and with the aid of the parity property of Legendre polynomials, it is found that for $z \leq 0$,

$$
\frac{P_{n}(\mu)}{R^{n+1}}=\frac{(-1)^{n}}{n !} \int_{0}^{\infty} J_{0}(\lambda r) e^{\lambda z} \lambda^{n} d \lambda .
$$

Using this, the displacement potential (13) can be rewritten as

$$
\frac{\phi_{0}}{T}=\int_{0}^{\infty} \omega_{1}(\lambda) J_{0}(\lambda r) e^{\lambda z} d \lambda, \quad \frac{\phi_{3}}{T}=\int_{0}^{\infty} \lambda \omega_{2}(\lambda) J_{0}(\lambda r) e^{\lambda z} d \lambda,
$$

where

$$
\omega_{1}(\lambda)=\sum_{n=0}^{\infty} A_{n}(-1)^{n} \lambda^{n} / n !, \quad \omega_{2}(\lambda)=\sum_{n=0}^{\infty} B_{n}(-1)^{n} \lambda^{n-1} / n ! .
$$

At this point, the expressions for stress and the surface divergence of the surface stress on the plane boundary can be obtained readily by substituting potentials $\left(12^{*}\right),\left(13^{*}\right)$, and (14) into (3) and (7) (see Equations (23) and (24)). By satisfying the plane boundary conditions (9), we get the relations

$$
\begin{array}{r}
\int_{0}^{\infty} \lambda^{2}\left(\left(\psi_{1}+(-c \lambda+1-2 v) \psi_{2}\right) e^{c \lambda}-\left(\omega_{1}+(-c \lambda-1+2 v) \omega_{2}\right) e^{-c \lambda}\right) J_{1}(\lambda r) d \lambda \\
=-\int_{0}^{\infty} \chi_{0} \lambda^{3}\left(\left(\psi_{1}-c \lambda \psi_{2}\right) e^{c \lambda}+\left(\omega_{1}-c \lambda \omega_{2}\right) e^{-c \lambda}\right) J_{1}(\lambda r) d \lambda \\
\int_{0}^{\infty} \lambda^{2}\left(\left(\psi_{1}+(-c \lambda+2-2 v) \psi_{2}\right) e^{c \lambda}+\left(\omega_{1}+(-c \lambda-2+2 v) \omega_{2}\right) e^{-c \lambda}\right) J_{0}(\lambda r) d \lambda \\
=0 .
\end{array}
$$


This is satisfied identically if

$$
\begin{gathered}
\left(\chi_{f 0} \lambda+1\right) \psi_{1}-\left(\chi_{f 0} c \lambda^{2}+c \lambda-1+2 \nu\right) \psi_{2} \\
=\left(1-\chi_{f 0} \lambda\right) \omega_{1} e^{-2 c \lambda}+\left(\chi_{f 0} c \lambda^{2}-c \lambda-1+2 v\right) \omega_{2} e^{-2 c \lambda}, \\
\psi_{1}+(-c \lambda+2-2 v) \psi_{2}=-\omega_{1} e^{-2 c \lambda}-(-c \lambda-2+2 v) \omega_{2} e^{-2 c \lambda} .
\end{gathered}
$$

Solving this for $\psi_{1}(\lambda)$ and $\psi_{2}(\lambda)$, we obtain

$$
\begin{array}{r}
\begin{array}{r}
\psi_{1}(\lambda)=-\left(\left(2\left(c+b_{1}\right) \lambda-(3-4 v)\right) \omega_{1}+\left(4(1-2 v)(1-v)-2 c\left(c+2 b_{1}\right) \lambda^{2}\right) \omega_{2}\right) \\
\times e^{-2 c \lambda} /\left(1+2 b_{1} \lambda\right),
\end{array} \\
\psi_{2}(\lambda)=-\left(2 \omega_{1}-\left((3-4 v)+2\left(c+b_{1}\right) \lambda\right) \omega_{2}\right) e^{-2 c \lambda} /\left(1+2 b_{1} \lambda\right),
\end{array}
$$

where $b_{1}=(1-v) \chi_{f 0}$. These are the conditions necessary to satisfy Equation (9). The stress components on the plane boundary are reduced to (25) by substituting Equations (16) and (17) in (23).

The next task is to satisfy the boundary conditions on the matrix/inhomogeneity interface. For this purpose, we must transform the potential (14) into spherical coordinates. From [Morse and Feshbach 1953, p. 1318], it is found that for $z \geq 0$,

$$
e^{\lambda z} J_{0}(\lambda r)=\sum_{n=0}^{\infty} \frac{(\lambda R)^{n}}{n !} P_{n}(\mu) .
$$

Replacing $(z)$ with $(-z),(\mu)$ with $(-\mu)$, and with the aid of the parity property of Legendre polynomials, it is deduced that for $z \leq 0$,

$$
e^{-\lambda z} J_{0}(\lambda r)=\sum_{n=0}^{\infty}(-1)^{n} \frac{(\lambda R)^{n}}{n !} P_{n}(\mu) .
$$

With the use of this equation, the displacement potential (14) can be expressed in spherical coordinates as

$$
\frac{\phi_{0}}{T}=\sum_{n=0}^{\infty} \alpha_{n} R^{n} P_{n}(\mu), \quad \frac{\phi_{3}}{T}=\sum_{n=0}^{\infty} \beta_{n} R^{n} P_{n}(\mu),
$$

where

$$
\alpha_{n}=\int_{0}^{\infty} \frac{(-1)^{n} \lambda^{n}}{n !} \psi_{1}(\lambda) d \lambda, \quad \beta_{n}=\int_{0}^{\infty} \frac{(-1)^{n} \lambda^{n}}{n !} \lambda \psi_{2}(\lambda) d \lambda .
$$

Substituting (16) and (17) into these two equations, expanding $1 /\left(1+2 b_{1} \lambda\right)$ in powers of $\lambda$ for small $b_{1}$, and using Euler's integral of the second kind

$$
\int_{0}^{\infty} \lambda^{n} e^{-c \lambda} d \lambda=n ! / c^{n+1} \quad(n, c>0),
$$


(see [Gradshteyn and Ryzhik 1994, p. 357]), we can write

$$
\alpha_{n}=\sum_{m=0}^{\infty}\left(f_{m}^{n} A_{m}+g_{m}^{n} B_{m}\right), \quad \beta_{n}=\sum_{m=0}^{\infty}\left(2(m+1) q_{m+1}^{n} A_{m}+h_{m}^{n} B_{m}\right),
$$

where

$$
\begin{aligned}
q_{m}^{n} & =\frac{(-1)^{m+n}}{m ! n !} \int_{0}^{\infty} \frac{\lambda^{m+n} e^{-2 c \lambda}}{\left(1+2 b_{1} \lambda\right)} d \lambda \\
& = \begin{cases}\frac{(-1)^{m+n}}{m ! n !} \frac{(m+n) !}{(2 c)^{m+n+1}} & \text { if } b_{1}=0, \\
\sum_{k=0}^{\infty}(-1)^{m+n+k} \frac{\left(2 b_{1}\right)^{k}(m+n+k) !}{m ! n !(2 c)^{m+n+k+1}} & \text { if } b_{1} \neq 0,\end{cases} \\
f_{m}^{n}=(3-4 v) q_{m}^{n}+2\left(c+b_{1}\right)(m+1) q_{m+1}^{n}, & \\
g_{m}^{n} & =4(1-2 v)(1-v) \frac{q_{m-1}^{n}}{m}-2 c\left(c+2 b_{1}\right)(m+1) q_{m+1}^{n}, \\
h_{m}^{n} & =(3-4 v) q_{m}^{n}-2\left(c+b_{1}\right)(m+1) q_{m+1}^{n} .
\end{aligned}
$$

The expressions for displacement, stress, and the interface divergence of the interface stress on the matrix/inhomogeneity interface can be derived by substituting displacement potentials into Equations (4) and (8). The elastic fields in the matrix are obtained by superposing potentials (12), (13), and (14*), while those in inhomogeneity are given by (15). These expressions are listed in the Appendix. To satisfy the boundary condition (11), we must have

$$
\begin{aligned}
& \sum_{n=0}^{\infty}\left(\begin{array}{c}
s_{A_{1}} A_{n}+s_{B_{1}} B_{n-1}+s_{B_{2}} B_{n+1}+s_{\alpha_{1}} \alpha_{n}+s_{\beta_{1}} \beta_{n-1} \\
+s_{\beta_{2}} \beta_{n+1}-\Gamma^{-1}\left(s_{\bar{A}_{1}} \bar{A}_{n}+s_{\bar{B}_{1}} \bar{B}_{n-1}+s_{\bar{B}_{2}} \bar{B}_{n+1}\right)
\end{array}\right) P_{n}(\mu) \\
& =-\frac{2(1-2 v) a}{3(1+v)} P_{0}(\mu)+\frac{2 a}{3} P_{2}(\mu), \\
& \sum_{n=1}^{\infty}\left(\begin{array}{c}
t_{A_{1}} A_{n}+t_{B_{1}} B_{n-1}+t_{B_{2}} B_{n+1}+t_{\alpha_{1}} \alpha_{n}+t_{\beta_{1}} \beta_{n-1} \\
+t_{\beta_{2}} \beta_{n+1}-\Gamma^{-1}\left(t_{\bar{A}_{1}} \bar{A}_{n}+t_{\bar{B}_{1}} \bar{B}_{n-1}+t_{\bar{B}_{2}} \bar{B}_{n+1}\right)
\end{array}\right) P_{n}^{\prime}(\mu)=-\frac{a}{3} P_{2}^{\prime}(\mu), \\
& \sum_{n=0}^{\infty}\left(\begin{array}{c}
\left(i_{s_{A_{1}}}+i_{A_{1}}\right) A_{n}+\left(i_{s_{B_{1}}}+i_{B_{1}}\right) B_{n-1}+\left(i_{s_{B_{2}}}+i_{B_{2}}\right) B_{n+1} \\
+\left(i_{s_{\alpha_{1}}}+i_{\alpha_{1}}\right) \alpha_{n}+\left(i_{s_{\beta_{1}}}+i_{\beta_{1}}\right) \beta_{n-1}+\left(i_{s_{\beta_{2}}}+i_{\beta_{2}}\right) \beta_{n+1} \\
+\left(\Gamma^{-1} i_{\bar{A}_{\bar{A}_{1}}}-i_{\bar{A}_{1}}\right) \bar{A}_{n}+\left(\Gamma^{-1} i_{\bar{B}_{\bar{B}_{1}}}-i_{\bar{B}_{1}}\right) \bar{B}_{n-1}+\left(\Gamma^{-1} i_{s_{\bar{B}_{2}}}-i_{\bar{B}_{2}}\right) \bar{B}_{n+1}
\end{array}\right) P_{n}(\mu) \\
& =\left(\frac{2 \tau_{0}}{a T}+\frac{4(1-2 v) \chi_{3}}{3 a(1+v)}-\frac{2}{3}\right) P_{0}(\mu)+\left(\frac{2 \chi_{3}}{3 a}+\frac{2}{3}\right) P_{2}(\mu),
\end{aligned}
$$




$$
\begin{gathered}
\sum_{n=1}^{\infty}\left(\begin{array}{c}
\left(l_{s_{A_{1}}}+l_{A_{1}}\right) A_{n}+\left(l_{s_{B_{1}}}+l_{B_{1}}\right) B_{n-1}+\left(l_{s_{B_{2}}}+l_{B_{2}}\right) B_{n+1} \\
+\left(l_{s_{\alpha_{1}}}+l_{\alpha_{1}}\right) \alpha_{n}+\left(l_{s_{\beta_{1}}}+l_{\beta_{1}}\right) \beta_{n-1}+\left(l_{s_{\beta_{2}}}+l_{\beta_{2}}\right) \beta_{n+1} \\
+\left(\Gamma^{-1} l_{s_{\bar{A}_{1}}}-l_{\bar{A}_{1}}\right) \bar{A}_{n}+\left(\Gamma^{-1} l_{s_{\bar{B}_{1}}}-l_{\bar{B}_{1}}\right) \bar{B}_{n-1}+\left(\Gamma^{-1} l_{s_{\bar{B}_{2}}}-l_{\bar{B}_{2}}\right) \bar{B}_{n+1}
\end{array}\right) P_{n}^{\prime}(\mu) \\
=\left(\frac{3 \chi_{0}-\chi_{1}}{3 a}-\frac{1}{3}\right) P_{2}^{\prime}(\mu),
\end{gathered}
$$

where $P_{n}^{\prime}(\mu)=d P_{n}(\mu) / d \mu, \Gamma=\bar{G} / G$, and the coefficients $s, t, i, l$ are defined in the Appendix (page 788). In Equations (19) and (20), the displacement containing $\alpha_{1}, \beta_{0}$ represents the rigid body motion of the matrix. We set $\alpha_{1}=\beta_{0}=0$ by viewing the matrix as a reference state. The displacement containing $\bar{A}_{1}, \bar{B}_{0}$ corresponds to the rigid body motion of the inhomogeneity. As a result, one of the two coefficients can be set to zero, so we set $\bar{A}_{1} \neq 0, \bar{B}_{0}=0$. Equating the coefficients of $P_{n}(\mu), P_{n}^{\prime}(\mu)$ on both sides of Equations (19)-(22), and with the aid of Equation (18), we obtain a set of linear algebraic equations leading to the unknown coefficients $\left\{A_{n}, B_{n}, \bar{A}_{n}, \bar{B}_{n}\right\}$.

\section{Results and discussion}

Results were obtained in order to illustrate the impact of surface and interface effects on the elastic field in the vicinity of the inhomogeneity (nanoparticle). The key parameters are the shear moduli ratio $\Gamma$ and the inhomogeneity position and size, denoted by $c$ and $a$, respectively. The shear modulus of the matrix is set to $G=26 \mathrm{GPa}$, while $\Gamma$ ranges over different values of the nanoparticle's stiffness. The limiting value $\Gamma=0$ represents a spherical void while $\Gamma \rightarrow \infty$ corresponds to a rigid particle. Poisson's ratios are kept equal $(v=\bar{v}=0.25)$ for the matrix and the inhomogeneity. The far-field biaxial tension is set at $T=100 \mathrm{MPa}$. Numerical computations indicate that the coefficients $A_{n}, B_{n}, \bar{A}_{n}$, and $\bar{B}_{n}$ decay monotonically with increasing $n$. These coefficients converge more rapidly for small ratios $a / c$. As a result, for all ratios $a / c$, less than 20 terms are necessary to obtain the elastic field, with an accuracy of five significant figures.

When surface and interface effects are ignored, the results are compared with the classical solution of a semi-infinite elastic body having a spherical cavity or a spherical inhomogeneity [Tsuchida and Nakahara 1970; Tsutsui et al. 1974], and were found to be in perfect agreement.

The impacts of interface and free-surface elasticity were studied separately. First, only the effects of interface elasticity were considered. The interface elastic constants were selected as $\lambda^{s}=6.85107 \mathrm{~N} / \mathrm{m}, \mu^{s}=-0.83145 \mathrm{~N} / \mathrm{m}$, and $\tau_{0}=0.91084 \mathrm{~N} / \mathrm{m}$ These quantities can be deduced from the manipulation of the surface properties of an aluminum [111] free surface in [Miller and Shenoy 2000]. 
4.1. Interface elasticity only: stress distribution. Normalized stress components with and without interface elasticity are shown in Figures 2-4. Stresses at three specific points of the matrix/inhomogeneity interface vary with the ratio $a / c$ (radius of the particle normalized by the distance from the free surface). The particle position is fixed at $c=50 \mathrm{~nm}$. It is clear that the stress changes significantly when the particle size is small, for both soft $(\Gamma=0.5)$ and hard $(\Gamma=2)$ particles. In some cases, the interface effects even change the sign of the stress for small ratios of $a / c$. As expected, the stress fields converge to the classical solutions when the inhomogeneity size increases.

Numerical calculations are also performed to study the impact of interface elasticity on the stress distribution at $R=a$ when a spherical particle of fixed size approaches the plane boundary. Results were similar to the ones illustrated in Figures 2-4. As long as the particle size is of nanometer size, interface elasticity significantly changes the particle/matrix interface stresses, even if the particle is pretty far from the plane boundary (free surface).

To examine the stress distribution along the interface, calculations were carried out for various shear moduli ratios (Figures 5-6). For the matrix, softer particles $(\Gamma<1)$ are affected more than harder particles $(\Gamma>1)$. As seen in Figure 6, top, the maximum shear stress $\tau_{1}$ converges to the classical solution as $\Gamma$ increases when the inhomogeneity is hard $(\Gamma>1)$, while it deviates greatly from the classical solution when the inhomogeneity is soft $(\Gamma<1)$.

The maximum principal stress $\bar{\sigma}_{1}$ is compressive, while its classical counterpart is tensile everywhere. The sign of $\bar{\sigma}_{1}$ has been altered by the interface effects. Unlike $\sigma_{1}, \bar{\sigma}_{1}$ is more sensitive to harder particles than to softer ones. Compared to $\bar{\sigma}_{1}, \bar{\tau}_{1}$ is only slightly affected by interface elasticity. It remains tensile at every point around the interface. The maximum variation of $\bar{\tau}_{1}$ from the classical solution occurs at $\varphi=\pi$ for soft inhomogeneity $(\Gamma<1)$.

Figure 7 illustrates the variation of stresses $\sigma_{r r}$ and $\sigma_{\theta \theta}$ with $r / c$, at the plane boundary. The impact of interface elasticity on $\sigma_{r r}$ and $\sigma_{\theta \theta}$ is analogous to that on $\sigma_{1}$ and $\tau_{1}$ : the softer the particle, the more significant the interface elasticity becomes. However, for large values of $r / c(\geq 3), \sigma_{r r}$ and $\sigma_{\theta \theta}$ converge to the classical solution.

By limit analysis, the solutions of the present problem with only the interface effects should approach those of a spherical inhomogeneity with interface effects in an infinite medium when $a \ll c$. With the ratio $a / c \leq 0.01$, the elastic field at the spherical interface is in agreement with the one obtained for the infinite medium, with an accuracy of at least five significant figures.

4.2. Free-surface elasticity only: stress distribution. Calculations were performed to determine the free-surface elasticity effects on the stress distribution at $z=-c$ 

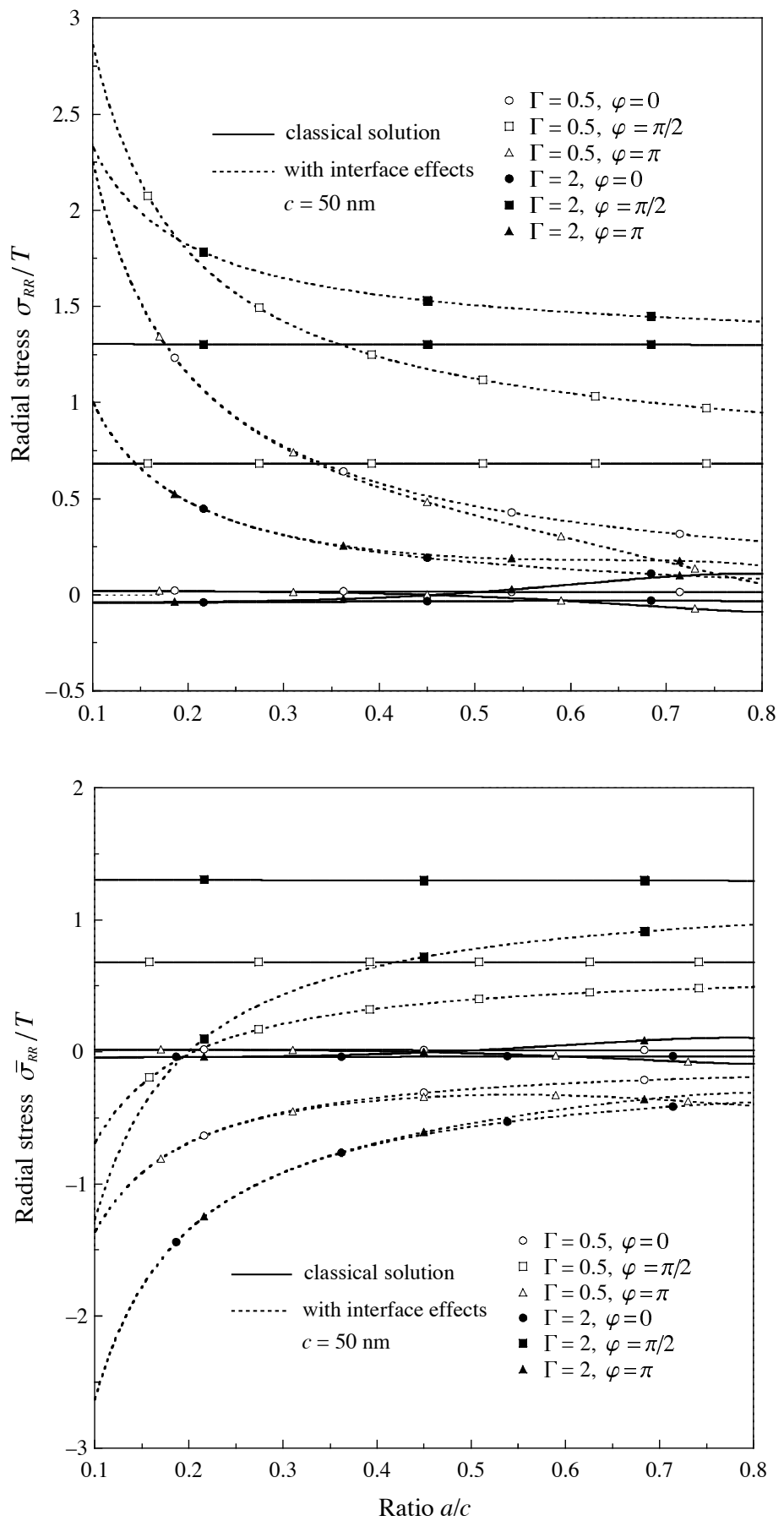

Figure 2. Variation of $\sigma_{R R}$ (top) and $\bar{\sigma}_{R R}$ (bottom) versus $a / c$ around the spherical interface at $\varphi=0, \pi / 2, \pi$. 

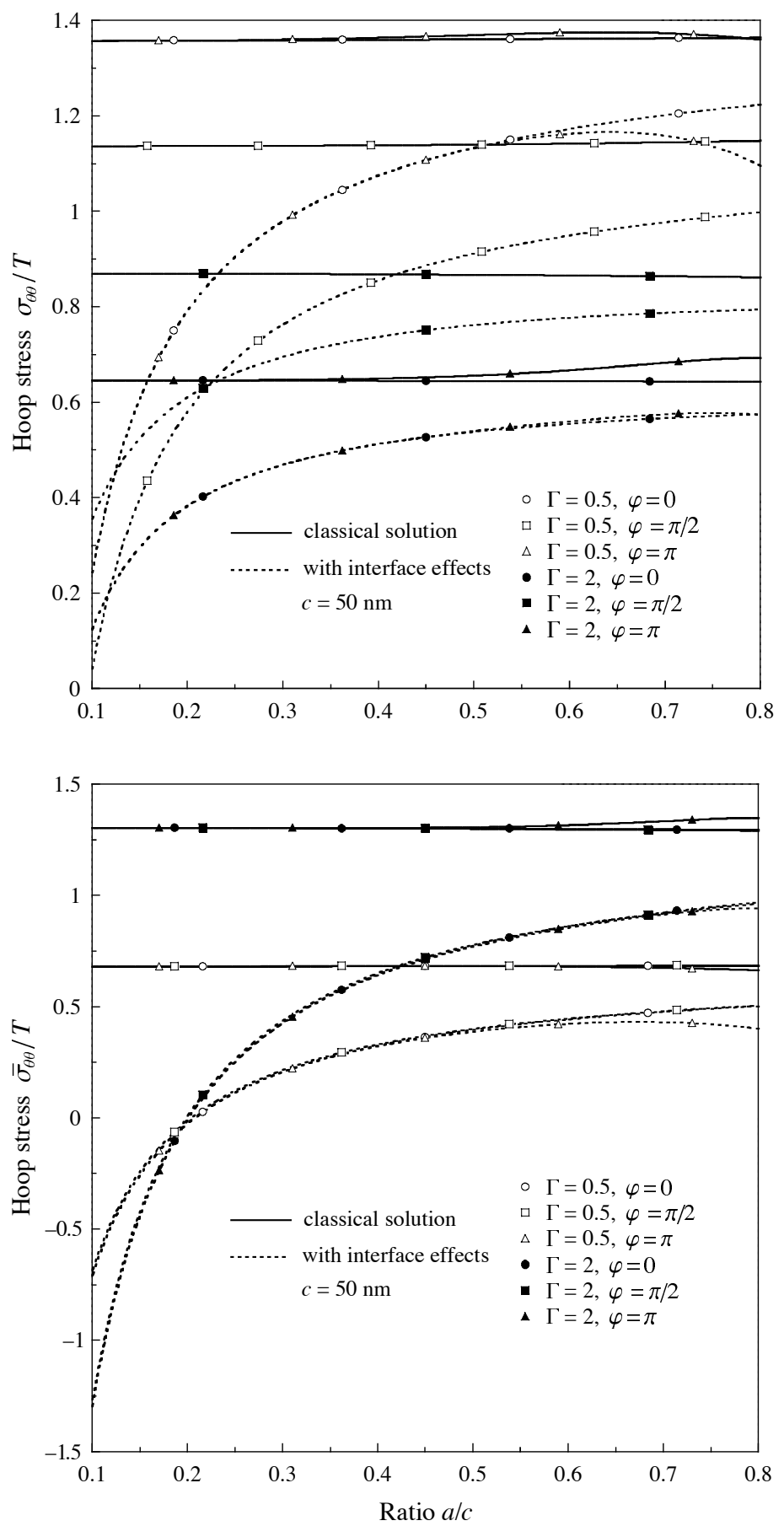

Figure 3. Variation of $\sigma_{\theta \theta}$ (top) and $\bar{\sigma}_{\theta \theta}$ (bottom) versus $a / c$ around the spherical interface at $\varphi=0, \pi / 2, \pi$. 

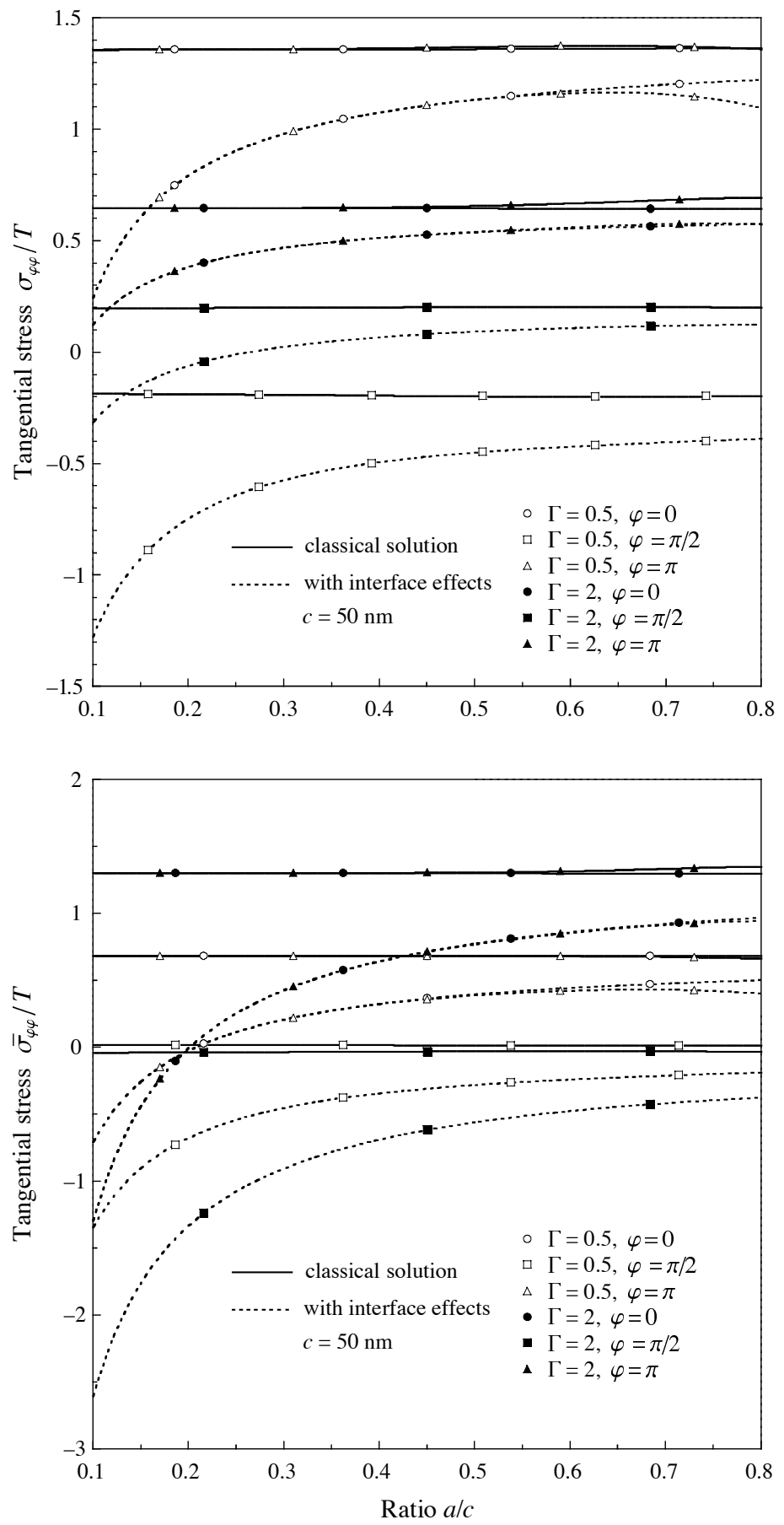

Figure 4. Variation of $\sigma_{\varphi \varphi}$ (top) and $\bar{\sigma}_{\varphi \varphi}$ (bottom) versus $a / c$ around the spherical interface at $\varphi=0, \pi / 2, \pi$. 

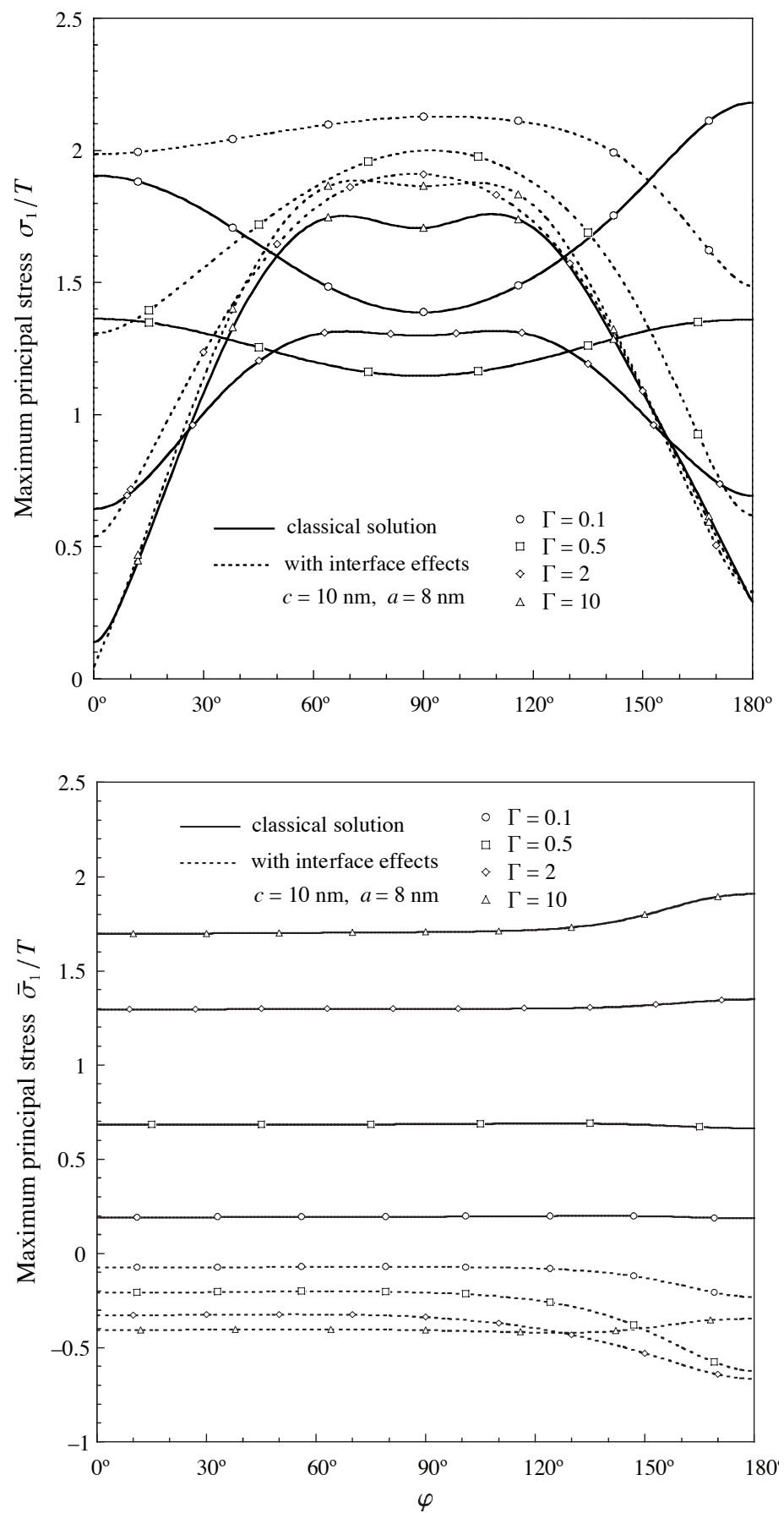

Figure 5. Variation of maximum principal stress $\sigma_{1}$ (top) and $\bar{\sigma}_{1}$ (bottom) with $\varphi$ at the spherical interface. 

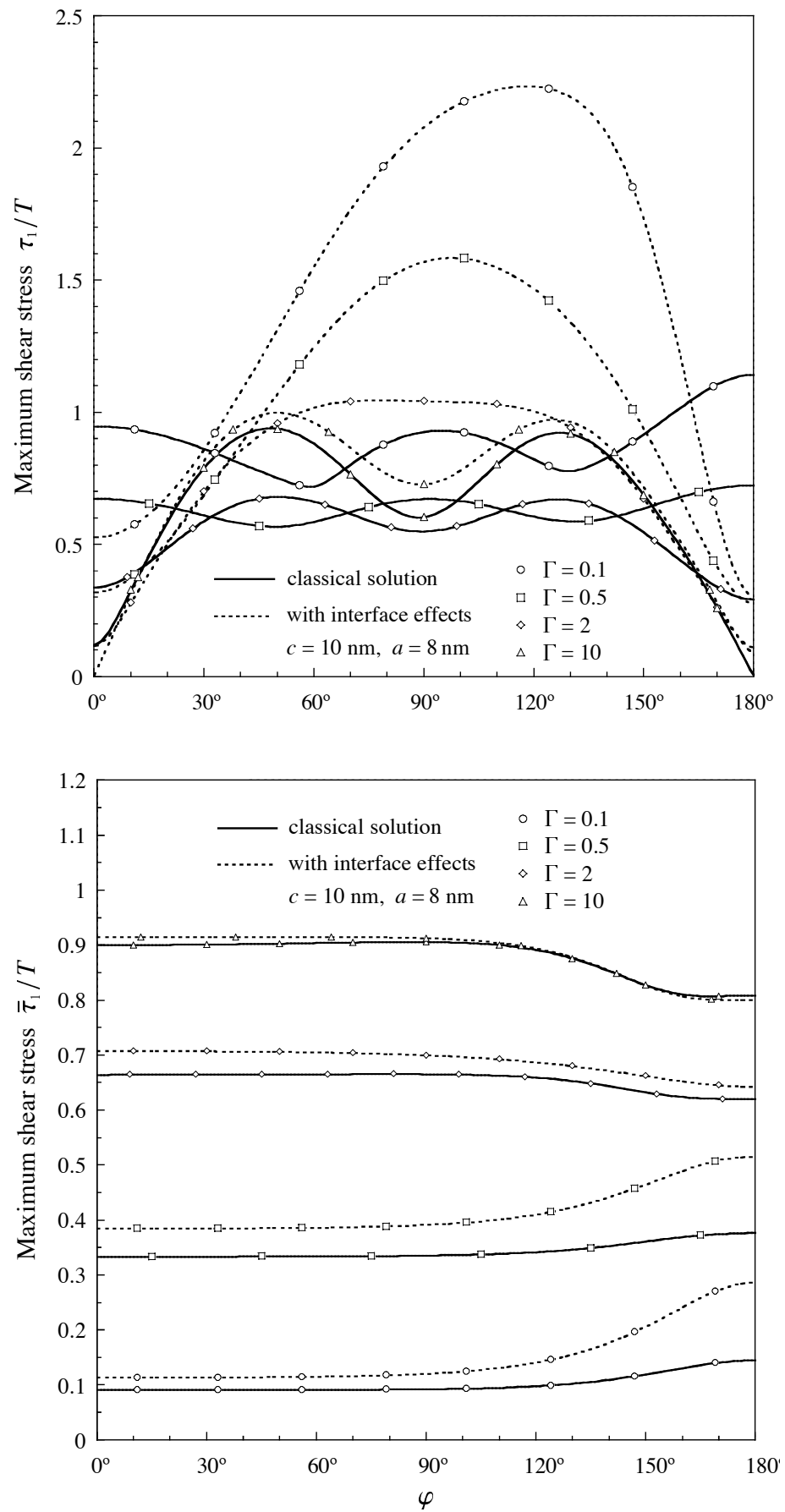

Figure 6. Variation of maximum shear stress $\tau_{1}$ (top) and $\bar{\tau}_{1}$ (bottom) with $\varphi$ at the spherical interface. 

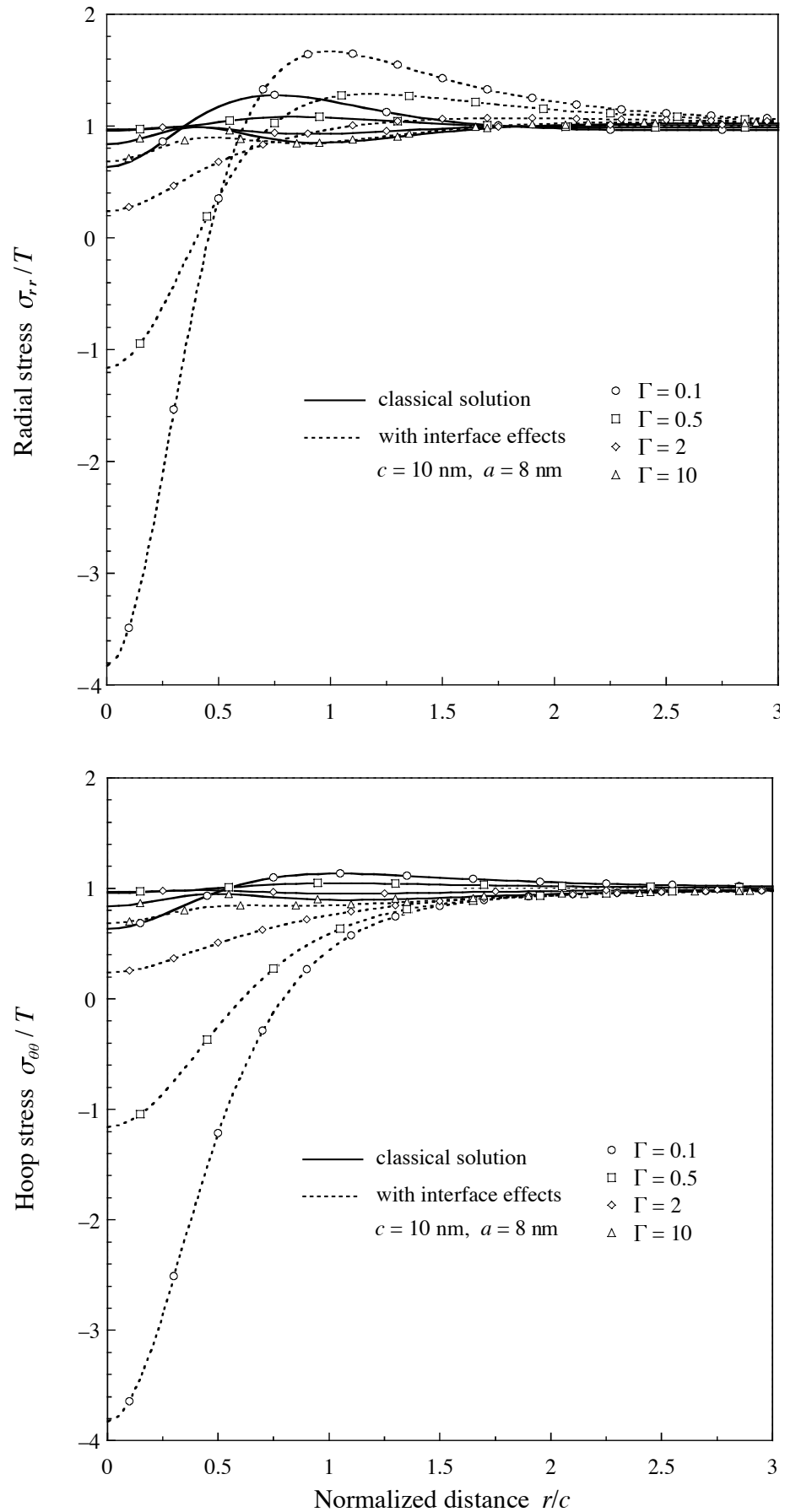

Figure 7. Variation of $\sigma_{r r}$ (top) and $\sigma_{\theta \theta}$ (bottom) with $r / c$ at the plane boundary. 




Figure 8. Variation of $\sigma_{r z}$ with $r / c$ at the plane boundary.

and $R=a$. We consider an aluminum [100] free surface with properties $\lambda^{f s}=$ $3.49387 \mathrm{~N} / \mathrm{m}, \mu^{f s}=-5.70915 \mathrm{~N} / \mathrm{m}$, and $\tau_{f 0}=0.56893 \mathrm{~N} / \mathrm{m}$ [Miller and Shenoy 2000]. The most important character of the plane surface effects is the nonvanishing shear stress $\sigma_{r z}$ at the plane boundary, as shown analytically in (25). In the classical solution, the conditions of zero traction at the plane boundary force $\sigma_{r z}$ to vanish. Figure 8 shows the distribution of $\sigma_{r z}$ with the normalized radial coordinate $r / c$, for $c=10 \mathrm{~nm}, a=8 \mathrm{~nm}$, and various shear moduli ratios. When the particle stiffness is not too different from the surrounding material $(\Gamma=0.5-2)$, surface elasticity is not important. Otherwise $(\Gamma=0.1,10)$, its influence is significant. Nevertheless, the magnitude of $\sigma_{r z}$ is small $(\leq 2 \% T)$. The impact of the free surface effects on $\sigma_{r r}$ and $\sigma_{\theta \theta}$ is found to be of the same order as that of $\sigma_{r z}$. The influence on the stress fields at the matrix/inhomogeneity interface is even smaller. One can safely conclude that compared to the applied tension at infinity, the free surface elasticity may be neglected.

\section{Summary and conclusions}

The axisymmetric problem of a spherical particle near the surface of a semi-infinite elastic body was solved, incorporating the effects of surface/interface elasticity. 
The solution was obtained within the framework of linear elasticity, through a displacement potential formulation.

The free surface and the interface were modeled as two-dimensional continuous spaces with vanishing thickness. Aside from the bulk material properties, the surface and interface boundaries have their own material constants (for example, the residual surface stress and surface Lamé constants). Numerical calculations indicate that the stress distribution along the plane boundary and the matrix/particle interface is significantly affected by surface/interface elasticity.

Following the analysis, one can make a few important observations:

- The impact of interface elasticity is a function of the particle size: the smaller the particle, the more important interface elasticity becomes. The elastic fields converge to the classical solution as the particle size increases (typically for $a \geq 100 \mathrm{~nm}$ ).

- The influence of interface elasticity depends on the softness or rigidity of the particles. For the matrix, the interface effects on the stress distribution are more pronounced when the particles are soft. However, the opposite is true for the particles themselves.

- In contrast with the zero traction requirement along the free surface in the classical solution, plane elasticity yields a nonvanishing shear stress, as shown in (25) and Figure 8. The plane surface effects become more important when the particle approaches the plane boundary. However, for distances where continuum theory is considered valid (typically $\geq 5 \mathrm{~nm}$ ), the magnitude of the disturbance due to the plane surface elasticity is small, when compared to the applied load.

- Due to the presence of the residual interface stress (see [He and $\mathrm{Li}$ in press]), superposition will not be valid when one considers interface elasticity. This is critical, because it significantly complicates the problem when multiple loads (say, misfit strain and mechanical load) are applied separately.

The study presented here illustrates that models incorporating nanoparticles near surfaces cannot ignore surface and particularly, interface elasticity effects. This is especially true when one models self-organized adatom clusters and islands, since most of these structures are of nanoscale size. The important problem of strained islands due to misfit strains will be addressed in a separate communication. 


\section{Appendix}

Elastic fields at the plane boundary. The stresses along the plane boundary are given below in terms of potentials $\left(12^{*}\right),\left(13^{*}\right)$, and (14):

$$
\begin{aligned}
\left(\frac{\sigma_{r r}}{T}\right)_{z=-c}=1 & -\int_{0}^{\infty} \lambda\left(\omega_{1}-c \lambda \omega_{2}\right)\left(\lambda J_{0}(\lambda r)-\frac{1}{r} J_{1}(\lambda r)\right) e^{-c \lambda} d \lambda \\
& -\int_{0}^{\infty} 2 v \lambda^{2} \omega_{2} J_{0}(\lambda r) e^{-c \lambda} d \lambda \\
& -\int_{0}^{\infty} \lambda\left(\psi_{1}-c \lambda \psi_{2}\right)\left(\lambda J_{0}(\lambda r)-\frac{1}{r} J_{1}(\lambda r)\right) e^{c \lambda} d \lambda \\
& +\int_{0}^{\infty} 2 v \lambda^{2} \psi_{2} J_{0}(\lambda r) e^{c \lambda} d \lambda, \\
\left(\frac{\sigma_{\theta \theta}}{T}\right)_{z=-c}=1 & -\int_{0}^{\infty} \lambda\left(\frac{1}{r}\left(\omega_{1}-c \lambda \omega_{2}\right) J_{1}(\lambda r)+2 v \lambda \omega_{2} J_{0}(\lambda r)\right) e^{-c \lambda} d \lambda \\
& -\int_{0}^{\infty} \lambda\left(\frac{1}{r}\left(\psi_{1}-c \lambda \psi_{2}\right) J_{1}(\lambda r)-2 v \lambda \psi_{2} J_{0}(\lambda r)\right) e^{c \lambda} d \lambda, \\
\left(\frac{\sigma_{z z}}{T}\right)_{z=-c}= & \int_{0}^{\infty} \lambda^{2}\left(\omega_{1}-(c \lambda+2-2 v) \omega_{2}\right) J_{0}(\lambda r) e^{-c \lambda} d \lambda \\
& +\int_{0}^{\infty} \lambda^{2}\left(\psi_{1}+(-c \lambda+2-2 v) \psi_{2}\right) J_{0}(\lambda r) e^{c \lambda} d \lambda, \\
\left(\frac{\sigma_{r z}}{T}\right)_{z=-c}= & -\int_{0}^{\infty} \lambda^{2}\left(\omega_{1}-(c \lambda+1-2 v) \omega_{2}\right) J_{1}(\lambda r) e^{-c \lambda} d \lambda \\
& +\int_{0}^{\infty} \lambda^{2}\left(\psi_{1}+(-c \lambda+1-2 v) \psi_{2}\right) J_{1}(\lambda r) e^{c \lambda} d \lambda .
\end{aligned}
$$

The surface divergence of the surface stress on the plane boundary is

$$
\begin{aligned}
\gamma_{r}^{f}=\chi_{f 0} T\left(\int_{0}^{\infty} \lambda^{3}\left(\omega_{1}-c \lambda \omega_{2}\right) J_{1}(\lambda r) e^{-c \lambda} d \lambda\right. & \\
& \left.+\int_{0}^{\infty} \lambda^{3}\left(\psi_{1}-c \lambda \psi_{2}\right) J_{1}(\lambda r) e^{c \lambda} \partial \lambda\right) .
\end{aligned}
$$

With Equations (16) and (17), Equation (23) is reduced to

$$
\begin{aligned}
\left(\frac{\sigma_{r r}}{T}\right)_{z=-c}=1 & +\sum_{n=0}^{\infty}(-1)^{n} A_{n}\left(-4 \frac{F_{0}^{n+2}}{n !}+4(1-v) \frac{1}{r} \frac{F_{1}^{n+1}}{n !}\right) \\
& +\sum_{n=0}^{\infty}(-1)^{n} B_{n}\left(4(1-2 v) \frac{F_{0}^{n+1}}{n !}+4 c \frac{F_{0}^{n+2}}{n !}\right. \\
& \left.-4(1-2 v)(1-v) \frac{1}{r} \frac{F_{1}^{n}}{n !}-4(1-v) c \frac{1}{r} \frac{F_{1}^{n+1}}{n !}\right), \\
\left(\frac{\sigma_{\theta \theta}}{T}\right)_{z=-c}=1 & +\sum_{n=0}^{\infty}(-1)^{n} A_{n}\left(-4 v \frac{F_{0}^{n+2}}{n !}-\frac{4(1-v)}{r} \frac{F_{1}^{n+1}}{n !}\right) \\
& +\sum_{n=0}^{\infty}(-1)^{n} B_{n}\left(4 v(1-2 v) \frac{F_{0}^{n+1}}{n !}+4 v c \frac{F_{0}^{n+2}}{n !}\right. \\
& \left.+\frac{4(1-2 v)(1-v)}{r} \frac{F_{1}^{n}}{n !}+\frac{4 c(1-v)}{r} \frac{F_{1}^{n+1}}{n !}\right),
\end{aligned}
$$




$$
\begin{aligned}
\left(\frac{\sigma_{r z}}{T}\right)_{z=-c} & =\sum_{n=0}^{\infty} 4 b_{1}(-1)^{n}\left(-A_{n} \frac{F_{1}^{n+3}}{n !}+B_{n}\left((1-2 v) \frac{F_{1}^{n+2}}{n !}+c \frac{F_{1}^{n+3}}{n !}\right)\right), \\
\left(\sigma_{z z}\right)_{z}=-c & =0
\end{aligned}
$$

where

$$
F_{v}^{n}=\int_{0}^{\infty} \frac{J_{v}(\lambda r) e^{-c \lambda} \lambda^{n}}{1+2 b_{1} \lambda} d \lambda
$$

With the aid of the Taylor's series expansion of $1 /\left(1+2 b_{1} \lambda\right)$ for small parameter $b_{1}$, and the relation

$$
\int_{0}^{\infty} J_{v}(\lambda r) e^{-c \lambda} \lambda^{n} d \lambda=\frac{(n-v) !}{\left(r^{2}+c^{2}\right)^{(n+1) / 2}} P_{n}^{v}\left(\frac{c}{\sqrt{r^{2}+c^{2}}}\right) \quad(c>0, v+n>-1)
$$

from [Gradshteyn and Ryzhik 1994, p. 732-733], Equation (27) can be approximated as

$$
F_{v}^{n} \approx \sum_{m=0}^{\infty}(-1)^{m}\left(2 b_{1}\right)^{m} \frac{(m+n+v) !}{\left(r^{2}+c^{2}\right)^{(m+n+1) / 2}} P_{m+n}^{v}\left(\frac{c}{\sqrt{r^{2}+c^{2}}}\right),
$$

where $P_{n}^{v}(\mu)$ is the associated Legendre function of order $n$ and degree $v$.

The length parameters in (6) are defined as

$$
\chi_{f 0}=\left(\lambda^{f s}+2 \mu^{f s}\right) / 4 G, \quad \chi_{f 1}=\left(\lambda^{f s}+\tau_{f 0}\right) / 4 G, \quad \chi_{f 2}=\chi_{f 0}-\chi_{f 1},
$$

where $\lambda^{f s}, \mu^{f s}$ denote the surface Lamé moduli for the plane boundary $(z=-c)$.

Elastic fields at the spherical interface. The displacements and stresses in the matrix at $R=a$ are given by

$$
\begin{aligned}
& \left(\frac{2 G u_{R}}{T}\right)_{R=a}=\sum_{n=0}^{\infty}\left(\frac{2}{3} a\left(\frac{1-2 v}{1+v} \delta_{0 n}-\delta_{2 n}\right)+s_{A_{1}} A_{n}+s_{B_{1}} B_{n-1}+s_{B_{2}} B_{n+1}\right. \\
& \left.+s_{\alpha_{1}} \alpha_{n}+s_{\beta_{1}} \beta_{n-1}+s_{\beta_{2}} \beta_{n+1}\right) P_{n}(\mu), \\
& \left(\frac{2 G u_{\varphi}}{T}\right)_{R=a}=\sqrt{1-\mu^{2}} \sum_{n=1}^{\infty}\left(\frac{1}{3} a \delta_{2 n}+t_{A_{1}} A_{n}+t_{B_{1}} B_{n-1}+t_{B_{2}} B_{n+1}\right. \\
& \left.+t_{\alpha_{1}} \alpha_{n}+t_{\beta_{1}} \beta_{n-1}+t_{\beta_{2}} \beta_{n+1}\right) P_{n}^{\prime}(\mu), \\
& \left(\frac{\sigma_{R R}}{T}\right)_{R=a}=\sum_{n=0}^{\infty}\left(\frac{2}{3}\left(\delta_{0 n}-\delta_{2 n}\right)+i_{A_{1}} A_{n}+i_{B_{1}} B_{n-1}+i_{B_{2}} B_{n+1}\right. \\
& \left.+i_{\alpha_{1}} \alpha_{n}+i_{\beta_{1}} \beta_{n-1}+i_{\beta_{2}} \beta_{n+1}\right) P_{n}(\mu) \text {, } \\
& \left(\frac{\sigma_{R \varphi}}{T}\right)_{R=a}=\sqrt{1-\mu^{2}} \sum_{n=1}^{\infty}\left(\frac{1}{3} \delta_{2 n}+l_{A_{1}} A_{n}+l_{B_{1}} B_{n-1}+l_{B_{2}} B_{n+1}\right. \\
& \left.+l_{\alpha_{1}} \alpha_{n}+l_{\beta_{1}} \beta_{n-1}+l_{\beta_{2}} \beta_{n+1}\right) P_{n}^{\prime}(\mu),
\end{aligned}
$$




$$
\begin{aligned}
& \left(\frac{\sigma_{\theta \theta}}{T}\right)_{R=a}=\sum_{n=1}^{\infty}\left(-\frac{A_{n}}{a^{n+3}}-\frac{B_{n-1}(n-1)(1-2 v)}{(2 n-1) a^{n+1}}-\frac{\beta_{n-1} a^{n-2}(n-1)(1-2 v)}{(2 n-1)}\right. \\
& -\frac{B_{n+1}((n+2)(1-2 v)+2(2 n+3) v)}{(2 n+3) a^{n+3}}-\alpha_{n} a^{n-2} \\
& \left.-\frac{\beta_{n+1} a^{n}(2(2 n+3) v+(n+2)(1-2 v))}{(2 n+3)}\right) \mu P_{n}^{\prime}(\mu) \\
& +\sum_{n=0}^{\infty}\left(\delta_{0 n}-\frac{A_{n}(n+1)}{a^{n+3}}-\frac{B_{n-1} n^{2}(1-2 \nu)}{(2 n-1) a^{n+1}}+\frac{\beta_{n-1} a^{n-2} n(n-1)(1-2 v)}{(2 n-1)}\right. \\
& -\frac{B_{n+1}(n+1)((n+2)(1-2 v)+2(2 n+3) v)}{(2 n+3) a^{n+3}}+\alpha_{n} a^{n-2} n \\
& \left.+\frac{\beta_{n+1} a^{n}(n+1)((n+1)(1-2 v)-2(2 n+3) v)}{(2 n+3)}\right) P_{n}(\mu) \text {, }
\end{aligned}
$$

$$
\begin{gathered}
\left(\frac{\sigma_{\varphi \varphi}}{T}\right)_{R=a}=\sum_{n=1}^{\infty}\left(\frac{A_{n}}{a^{n+3}}+\frac{B_{n-1}(n-1)(3-2 v)}{(2 n-1) a^{n+1}}+\frac{\beta_{n-1} a^{n-2}(n-1)(3-2 v)}{(2 n-1)}\right. \\
-\frac{B_{n+1}(2(2 n+3)(1-v)-(n+2)(3-2 v))}{(2 n+3) a^{n+3}}+\alpha_{n} a^{n-2} \\
\left.-\frac{\beta_{n+1} a^{n}(2(2 n+3)(1-v)-(n+2)(3-2 v))}{(2 n+3)}\right) \mu P_{n}^{\prime}(\mu)
\end{gathered}
$$$$
+\sum_{n=0}^{\infty}\left(\frac{1}{3}\left(\delta_{0 n}+2 \delta_{2 n}\right)-\frac{A_{n}(n+1)^{2}}{a^{n+3}}-\frac{B_{n-1} n^{2}(n-2 v)}{(2 n-1) a^{n+1}}\right.
$$$$
-\frac{B_{n+1}(n+1)((n+2)(n+2-2 v)+2(2 n+3)(1-v))}{(2 n+3) a^{n+3}}
$$$$
-\alpha_{n} a^{n-2} n^{2}-\frac{\beta_{n-1} a^{n-2}(n-1) n(n-1+2 v)}{(2 n-1)}
$$$$
\left.-\frac{\beta_{n+1} a^{n}(n+1)((n+1)(n+1+2 v)+2(2 n+3)(1-v))}{(2 n+3)}\right) P_{n}(\mu) \text {. }
$$

The displacements and stresses in the inhomogeneity at $R=a$ are given by $\left(\frac{2 \bar{G} \bar{u}_{R}}{T}\right)_{R=a}=\sum_{n=0}^{\infty}\left(s_{\bar{A}_{1}} \bar{A}_{n}+s_{\bar{B}_{1}} \bar{B}_{n-1}+s_{\bar{B}_{2}} \bar{B}_{n+1}\right) P_{n}(\mu)$, 


$$
\begin{aligned}
& \left(\frac{2 \bar{G} \bar{u}_{\varphi}}{T}\right)_{R=a}=\sqrt{1-\mu^{2}} \sum_{n=1}^{\infty}\left(t_{\bar{A}_{1}} \bar{A}_{n}+t_{\bar{B}_{1}} \bar{B}_{n-1}+t_{\bar{B}_{2}} \bar{B}_{n+1}\right) P_{n}^{\prime}(\mu), \\
& \left(\frac{\bar{\sigma}_{R R}}{T}\right)_{R=a}=\sum_{n=0}^{\infty}\left(i_{\bar{A}_{1}} \bar{A}_{n}+i_{\bar{B}_{1}} \bar{B}_{n-1}+i_{\bar{B}_{2}} \bar{B}_{n+1}\right) P_{n}(\mu), \\
& \left(\frac{\bar{\sigma}_{R \varphi}}{T}\right)_{R=a}=\sqrt{1-\mu^{2}} \sum_{n=1}^{\infty}\left(l_{\bar{A}_{1}} \bar{A}_{n}+l_{\bar{B}_{1}} \bar{B}_{n-1}+l_{\bar{B}_{2}} \bar{B}_{n+1}\right) P_{n}^{\prime}(\mu), \\
& \left(\frac{\bar{\sigma}_{\theta \theta}}{T}\right)_{R=a}=-\sum_{n=1}^{\infty}\left(\bar{A}_{n} a^{n-2}+\frac{\bar{B}_{n-1} a^{n-2}(n-1)(1-2 \bar{v})}{(2 n-1)}\right. \\
& \left.+\frac{\bar{B}_{n+1} a^{n}(2(2 n+3) \bar{v}+(n+2)(1-2 \bar{v}))}{(2 n+3)}\right) \mu P_{n}^{\prime}(\mu) \\
& +\sum_{n=0}^{\infty}\left(\bar{A}_{n} a^{n-2} n+\frac{\bar{B}_{n-1} a^{n-2} n(n-1)(1-2 \bar{v})}{(2 n-1)}\right. \\
& \left.+\frac{\bar{B}_{n+1} a^{n}(n+1)((n+1)(1-2 \bar{v})-2(2 n+3) \bar{v})}{(2 n+3)}\right) P_{n}(\mu), \\
& \left(\frac{\bar{\sigma}_{\varphi \varphi}}{T}\right)_{R=a}=\sum_{n=1}^{\infty}\left(\bar{A}_{n} a^{n-2}+\frac{\bar{B}_{n-1} a^{n-2}(n-1)(3-2 \bar{\nu})}{(2 n-1)}\right. \\
& \left.-\frac{\bar{B}_{n+1} a^{n}(2(2 n+3)(1-\bar{v})-(n+2)(3-2 \bar{v}))}{(2 n+3)}\right) \mu P_{n}^{\prime}(\mu) \\
& -\sum_{n=0}^{\infty}\left(\bar{A}_{n} a^{n-2} n^{2}+\frac{\bar{B}_{n-1} a^{n-2}(n-1) n(n-1+2 \bar{v})}{(2 n-1)}\right. \\
& \left.+\frac{\bar{B}_{n+1} a^{n}(n+1)((n+1)(n+1+2 \bar{v})+2(2 n+3)(1-\bar{v}))}{(2 n+3)}\right) P_{n}(\mu) .
\end{aligned}
$$

The interface divergence of the interface stress at $R=a$ is given by

$$
\begin{array}{r}
\gamma_{R}=T \sum_{n=0}^{\infty}\left(-\left(\frac{2}{a T} \tau_{0}+\frac{4(1-2 v) \chi_{3}}{3 a(1+v)}\right) \delta_{0 n}-\frac{2 \chi_{3}}{3 a} \delta_{2 n}+i_{S_{A_{1}}} A_{n}+i_{S_{B_{1}}} B_{n-1}+i_{S_{B_{2}}} B_{n+1}\right. \\
\left.+i_{S_{\alpha_{1}}} \alpha_{n}+i_{S_{\beta_{1}}} \beta_{n-1}+i_{S_{\beta_{2}}} \beta_{n+1}+\Gamma^{-1}\left(i_{S_{\bar{A}_{1}}} \bar{A}_{n}+i_{S_{\bar{B}_{1}}} \bar{B}_{n-1}+i_{S_{\bar{B}_{2}}} \bar{B}_{n+1}\right)\right) P_{n}(\mu), \\
\gamma_{\varphi}=T \sqrt{1-\mu^{2}} \sum_{n=1}^{\infty}\left(-\frac{\left(3 \chi_{0}-\chi_{1}\right)}{3 a} \delta_{2 n}+l_{S_{A_{1}}} A_{n}+l_{S_{B_{1}}} B_{n-1}+l_{S_{B_{2}}} B_{n+1}+l_{S_{\alpha_{1}}} \alpha_{n}\right. \\
\left.+l_{S_{\beta_{1}}} \beta_{n-1}+l_{S_{\beta_{2}}} \beta_{n+1}+\Gamma^{-1}\left(l_{S_{\bar{A}_{1}}} \bar{A}_{n}+l_{S_{\bar{B}_{1}}} \bar{B}_{n-1}+l_{S_{\bar{B}_{2}}} \bar{B}_{n+1}\right)\right) P_{n}^{\prime}(\mu) .
\end{array}
$$

In these equations, the coefficients are defined as 


$$
\begin{aligned}
& s_{A_{1}}=-\frac{(n+1)}{a^{n+2}}, \quad t_{A_{1}}=-\frac{1}{a^{n+2}}, \\
& s_{B_{1}}=-\frac{n(n+3-4 v)}{(2 n-1) a^{n}}, \quad t_{B_{1}}=-\frac{(n-4+4 v)}{(2 n-1) a^{n}}, \\
& s_{B_{2}}=-\frac{(n+1)(n+5-4 v)}{(2 n+3) a^{n+2}}, \quad t_{B_{2}}=-\frac{(n+5-4 v)}{(2 n+3) a^{n+2}}, \\
& s_{\alpha_{1}}=n a^{n-1}\left(1-\delta_{1 n}\right), \quad t_{\alpha_{1}}=-a^{n-1}\left(1-\delta_{1 n}\right), \\
& s_{\beta_{1}}=\frac{n(n-4+4 v) a^{n-1}\left(1-\delta_{1 n}\right)}{(2 n-1)}, \quad t_{\beta_{1}}=-\frac{(n-4+4 v) a^{n-1}\left(1-\delta_{1 n}\right)}{(2 n-1)} \text {, } \\
& s_{\beta_{2}}=\frac{(n+1)(n-2+4 v) a^{n+1}}{(2 n+3)}, \quad t_{\beta_{2}}=-\frac{(n+5-4 v) a^{n+1}}{(2 n+3)}, \\
& s_{\bar{A}_{1}}=n a^{n-1}, \quad t_{\bar{A}_{1}}=-a^{n-1}, \\
& s_{\bar{B}_{1}}=\frac{n(n-4+4 \bar{v}) a^{n-1}\left(1-\delta_{1 n}\right)}{(2 n-1)}, \quad t_{\bar{B}_{1}}=-\frac{(n-4+4 \bar{\nu}) a^{n-1}\left(1-\delta_{1 n}\right)}{(2 n-1)}, \\
& s_{\bar{B}_{2}}=\frac{(n+1)(n-2+4 \bar{v}) a^{n+1}}{(2 n+3)}, \quad t_{\bar{B}_{2}}=-\frac{(n+5-4 \bar{v}) a^{n+1}}{(2 n+3)}, \\
& i_{A_{1}}=\frac{(n+1)(n+2)}{a^{n+3}}, \\
& i_{B_{1}}=\frac{n\left(n^{2}+3 n-2 v\right)}{(2 n-1) a^{n+1}}, \\
& i_{B_{2}}=\frac{(n+1)(n+2)(n+5-4 v)}{(2 n+3) a^{n+3}}, \\
& i_{S_{A_{1}}}=\frac{(n+1)(n+2) \chi_{3}}{a^{n+4}}, \\
& i_{S_{B_{1}}}=\frac{n(n(n+1)-2(1-2 v)(n-1)) \chi_{3}}{(2 n-1) a^{n+2}}, \\
& i_{S_{B_{2}}}=\frac{(n+1)(n+2)(n+5-4 v) \chi_{3}}{(2 n+3) a^{n+4}}, \\
& i_{S_{\alpha_{1}}}=(n-1) n \chi_{3} a^{n-3} \text {, } \\
& i_{S_{\beta_{1}}}=\frac{(n-1) n(n-4+4 v) \chi_{3} a^{n-3}}{(2 n-1)}, \\
& i_{\beta_{2}}=\frac{(n+1)\left(n^{2}-n-2-2 v\right) a^{n}}{(2 n+3)}, \quad i_{S_{\beta_{2}}}= \\
& \frac{(n+1)(n(n+1)+2(1-2 v)(n+2)) \chi_{3} a^{n-1}}{(2 n+3)}, \\
& i_{\bar{A}_{1}}=(n-1) n a^{n-2}, \\
& i_{S_{\bar{A}_{1}}}=(n-1) n \chi_{3} a^{n-3} \text {, } \\
& i_{\bar{B}_{1}}=\frac{(n-1) n(n-4+4 \bar{v}) a^{n-2}}{(2 n-1)}, \quad i_{S_{\bar{B}_{1}}}=\frac{(n-1) n(n-4+4 \bar{\nu}) \chi_{3} a^{n-3}}{(2 n-1)} \text {, } \\
& i_{\bar{B}_{2}}=\frac{(n+1)\left(n^{2}-n-2-2 \bar{v}\right) a^{n}}{(2 n+3)}, \quad i_{S_{\bar{B}_{2}}}= \\
& \frac{(n+1)(n(n+1)+2(1-2 \bar{v})(n+2)) \chi_{3} a^{n-1}}{(2 n+3)}
\end{aligned}
$$




$$
\begin{aligned}
& l_{A_{1}}=\frac{(n+2)}{a^{n+3}}, \\
& l_{S_{A_{1}}}=\frac{(n+2)\left(n \chi_{0}+\chi_{1}\right)}{a^{n+4}}, \\
& \left(n^{3}-2 n^{2}(1-2 v)-2 n+4(1-v)\right) \chi_{0} \\
& l_{B_{1}}=\frac{\left(n^{2}-2+2 v\right)}{(2 n-1) a^{n+1}}, \\
& l_{S_{B_{1}}}=\frac{+\left(n^{2}+4(1-v)(n-1)\right) \chi_{1}}{(2 n-1) a^{n+2}} \text {, } \\
& l_{B_{2}}=\frac{(n+2)(n+5-4 v)}{(2 n+3) a^{n+3}}, \\
& l_{S_{B_{2}}}=\frac{(n+2)(n+5-4 v)\left(n \chi_{0}+\chi_{1}\right)}{(2 n+3) a^{n+4}}, \\
& l_{\alpha_{1}}=-(n-1) a^{n-2} \text {, } \\
& l_{S_{\alpha_{1}}}=(n-1)\left((n+1) \chi_{0}-\chi_{1}\right) a^{n-3} \text {, } \\
& l_{\beta_{1}}=-\frac{(n-1)(n-4+4 v) a^{n-2}}{(2 n-1)} \\
& l_{S_{\beta_{1}}}=\frac{(n-1)(n-4+4 v)\left((n+1) \chi_{0}-\chi_{1}\right) a^{n-3}}{(2 n-1)} \text {, } \\
& \left(\left(n^{3}+n^{2}(5-4 v)+n(5-8 v)-3\right) \chi_{0}\right. \\
& l_{\beta_{2}}=-\frac{\left(n^{2}+2 n-1+2 v\right) a^{n}}{(2 n+3)}, \\
& l_{S_{\beta_{2}}}=\frac{\left.-\left((n+1)^{2}-4(1-v)(n+2)\right) \chi_{1}\right) a^{n-1}}{(2 n+3)}, \\
& l_{\bar{A}_{1}}=-(n-1) a^{n-2} \text {, } \\
& l_{S_{\bar{A}_{1}}}=(n-1)\left((n+1) \chi_{0}-\chi_{1}\right) a^{n-3} \text {, } \\
& l_{\bar{B}_{1}}=-\frac{(n-1)(n-4+4 \bar{v}) a^{n-2}}{(2 n-1)} \text {, } \\
& l_{S_{\bar{B}_{1}}}=\frac{(n-1)(n-4+4 \bar{v})\left((n+1) \chi_{0}-\chi_{1}\right) a^{n-3}}{(2 n-1)}, \\
& l_{S_{\bar{B}_{2}}}=\frac{\left(\left(n^{3}+n^{2}(5-4 \bar{v})+n(5-8 \bar{v})-3\right) \chi_{0}\right.}{\left.-\left((n+1)^{2}-4(1-\bar{v})(n+2)\right) \chi_{1}\right) a^{n-1}} . \\
& l_{\bar{B}_{2}}=-\frac{\left(n^{2}+2 n-1+2 \bar{v}\right) a^{n}}{(2 n+3)},
\end{aligned}
$$

The length scale parameters are defined as

$$
\begin{aligned}
& \chi_{0}=\frac{1}{4 G}\left(\lambda^{S}+2 \mu^{S}\right), \quad \chi_{1}=\frac{1}{4 G}\left(\lambda^{S}+\tau_{0}\right), \\
& \chi_{2}=\chi_{0}-\chi_{1}, \quad \chi_{3}=\chi_{0}+\chi_{1},
\end{aligned}
$$

where $\lambda^{S}, \mu^{S}$ are the interface Lamé moduli for spherical interface $(R=a)$.

\section{References}

[Adamson 1982] A. W. Adamson, Physical chemistry of surfaces, Wiley, New York, 1982.

[Cahn and Larche 1982] J. W. Cahn and F. Larche, "Surface stress and the chemical equilibrium of small crystals. II. solid particles embedded in a solid matrix”, Acta Metall. 30:1 (1982), 51-56.

[Duan et al. 2005a] H. L. Duan, J. Wang, Z. P. Huang, and B. L. Karihaloo, "Eshelby formalism for nano-inhomogeneities", P. Roy. Soc. Lond. A Mat. 461:2062 (2005), 3335-3353.

[Duan et al. 2005b] H. L. Duan, J. Wang, Z. P. Huang, and Z. Y. Luo, "Stress concentration tensors of inhomogeneities with interface effects", Mech. Mater. 37:7 (2005), 723-736.

[Gradshteyn and Ryzhik 1994] I. S. Gradshteyn and I. M. Ryzhik, Table of integrals, series and products, 5th ed., Academic Press, San Diego, 1994. 
[Gurtin and Murdoch 1975a] M. E. Gurtin and A. I. Murdoch, "A continuum theory of elastic material surfaces", Arch. Ration. Mech. An. 57:4 (1975), 291-323.

[Gurtin and Murdoch 1975b] M. E. Gurtin and A. I. Murdoch, "A continuum theory of elastic material surfaces", Arch. Ration. Mech. An. 59:4 (1975), 389-390.

[Gurtin and Murdoch 1978] M. E. Gurtin and A. I. Murdoch, "Surface stress in solids", Int. J. Solids Struct. 14:6 (1978), 431-440.

[Gurtin et al. 1998] M. E. Gurtin, J. Weissmuller, and F. Larche, "A general theory of curved deformable interfaces in solids at equilibrium”, Philos. Mag. A 78:5 (1998), 1093-1109.

[Miller and Shenoy 2000] R. E. Miller and V. B. Shenoy, "Size-dependent elastic properties of nanosized structural elements", Nanotechnology 11:3 (2000), 139-147.

[Mindlin and Cheng 1950] R. D. Mindlin and D. H. Cheng, "Thermoelastic stress in the semi-infinite solid", J. Appl. Phys. 21:9 (1950), 931-933.

[Morse and Feshbach 1953] P. M. Morse and H. Feshbach, Methods of theoretical physics, vol. II, McGraw-Hill, New York, 1953.

[Mura 1987] T. Mura, Micromechanics of defects in solids, Martinus Nijhoff, The Hague, 1987.

[Sharma and Ganti 2004] P. Sharma and S. Ganti, "Size-dependent Eshelby's tensor for embedded nano-inclusions incorporating surface/interface energies", J. Appl. Mech. (Trans. ASME) 71:5 (2004), 663-671.

[Sharma et al. 2003] P. Sharma, S. Ganti, and N. Bhate, "Effect of surfaces on the size-dependent elastic state of nano-inhomogeneities", Appl. Phys. Lett. 82:4 (2003), 535-537.

[Sneddon 1951] I. N. Sneddon, Fourier transforms, McGraw-Hill, New York, 1951.

[Tersoff et al. 1996] J. Tersoff, C. Teichert, and M. G. Lagally, "Self-organization in growth of quantum dot superlattices", Phys. Rev. Lett. 76:10 (1996), 1675-1678.

[Tsuchida and Nakahara 1970] E. Tsuchida and I. Nakahara, "Three-dimensional stress concentration around a spherical cavity in a semi-infinite elastic body", Bull. Jpn. Soc. Mech. Eng. 13:58 (1970), 499-508.

[Tsuchida and Nakahara 1972] E. Tsuchida and I. Nakahara, "Stresses in a semi-infinite body subjected to uniform pressure on the surface of a cavity and the plane boundary", Bull. Jpn. Soc. Mech. Eng. 15:79 (1972), 1-10.

[Tsutsui et al. 1974] S. Tsutsui, S. Chikamatsu, and K. Saito, "Stress field of the semi-infinite medium containing a spherical inclusion under all-around tension", Trans. Jpn. Soc. Mech. Eng. 40:337 (1974), 2484-2493.

Received 23 Dec 2005.

Changwen Mi: mi@uwyo.edu

Department of Mechanical Engineering, University of Wyoming, Laramie WY 82071, United States

DEMITRIS A. KouRIS: kouris@uwyo.edu

Department of Mechanical Engineering, University of Wyoming, Laramie WY 82071, United States 